Article

\title{
Prebiotic Xylo-Oligosaccharides Ameliorate High-Fat-Diet-Induced Hepatic Steatosis in Rats
}

\author{
Sanna Lensu ${ }^{1} \mathbb{D}$, Raghunath Pariyani ${ }^{2}$, Elina Mäkinen ${ }^{1}$, Baoru Yang ${ }^{2}$, Wisam Saleem ${ }^{3}$, \\ Eveliina Munukka ${ }^{4,5}$, Maarit Lehti ${ }^{1}$, Anastasiia Driuchina ${ }^{1}$, Jere Lindén ${ }^{6}$, Marja Tiirola ${ }^{7}$, \\ Leo Lahti ${ }^{3}$ and Satu Pekkala ${ }^{1,5, *}$ \\ 1 Faculty of Sport and Health Sciences, University of Jyväskylä, FI-40014 Jyväskylä, Finland; \\ sanna.t.k.lensu@jyu.fi (S.L.); elina.e.makinen@jyu.fi (E.M.); maarit.t.lehti@jyu.fi (M.L.); \\ anastasiia.a.driuchina@jyu.fi (A.D.) \\ 2 Food Chemistry and Food Development, Department of Biochemistry, University of Turku, FI-20014 Turku, \\ Finland; raghunath.pariyani@utu.fi (R.P.); bayang@utu.fi (B.Y.) \\ 3 Department of Future Technologies, University of Turku, FI-20014 Turku, Finland; \\ wisam.tariqsaleem@utu.fi (W.S.); leo.lahti@utu.fi (L.L.) \\ 4 Institute of Biomedicine, University of Turku, FI-20014 Turku, Finland; laevmu@utu.fi \\ 5 Department of Clinical Microbiology, Turku University Hospital, FI-20521 Turku, Finland \\ 6 Veterinary Pathology and Parasitology, University of Helsinki, FIN-00014 Helsinki, Finland; \\ jere.linden@helsinki.fi \\ 7 Department of Environmental and Biological Sciences, University of Jyväskylä, FI-40014 Jyväskylä, Finland; \\ marja.tiirola@jyu.fi \\ * Correspondence: satu.p.pekkala@jyu.fi; Tel.: +358-45-358-28-98
}

Received: 7 September 2020; Accepted: 20 October 2020; Published: 22 October 2020

check for updates

\begin{abstract}
Understanding the importance of the gut microbiota (GM) in non-alcoholic fatty liver disease (NAFLD) has raised the hope for therapeutic microbes. We have shown that high hepatic fat content associated with low abundance of Faecalibacterium prausnitzii in humans and, further, the administration of F. prausnitzii prevented NAFLD in mice. Here, we aimed at targeting F. prausnitzii by prebiotic xylo-oligosaccharides (XOS) to treat NAFLD. First, the effect of XOS on F. prausnitzii growth was assessed in vitro. Then, XOS was supplemented or not with high (HFD, $60 \%$ of energy from fat) or low (LFD) fat diet for 12 weeks in Wistar rats ( $n=10 /$ group). XOS increased F. prausnitzii growth, having only a minor impact on the GM composition. When supplemented with HFD, XOS ameliorated hepatic steatosis. The underlying mechanisms involved enhanced hepatic $\beta$-oxidation and mitochondrial respiration. Nuclear magnetic resonance $\left({ }^{1} \mathrm{H}-\mathrm{NMR}\right)$ analysis of cecal metabolites showed that, compared to the HFD, the LFD group had a healthier cecal short-chain fatty acid profile and on the HFD, XOS reduced cecal isovalerate and tyrosine, metabolites previously linked to NAFLD. Cecal branched-chain fatty acids associated positively and butyrate negatively with hepatic triglycerides. In conclusion, XOS supplementation can ameliorate NAFLD by improving hepatic oxidative metabolism and affecting GM.
\end{abstract}

Keywords: prebiotic; oligosaccharides; gut microbiota; fatty liver; metabolism; mitochondria

\section{Introduction}

In western countries, around $30 \%$ of the normal weight and up to $90 \%$ of the obese population are estimated to suffer from non-alcoholic fatty liver disease (NAFLD) [1]. NAFLD is defined as excessive fat accumulation in the liver without the patient drinking excessive alcohol or using steatogenic drugs. NAFLD can be categorized into simple hepatic steatosis, which is diagnosed as the presence of fat 
accumulation without histological or biochemical injuries, and non-alcoholic steatohepatitis (NASH), which is characterized by hepatic steatosis, inflammation and damage of the hepatocytes [2,3].

Increasing evidence shows that the pathogenesis of NAFLD is associated with environmental, host genetic and physiological factors [4], such as increased lipid storage [5-7] and mitochondrial dysfunction [7]. Frequently, dietary factors and excessive caloric intake are involved in the pathogenesis of NAFLD, and they are also important determinants of the gut microbiota (GM) composition of the host [8]. The GM refers to the trillions of tiny microbial cells inhabiting the gastrointestinal tract that break down the macromolecules and nutrients from the ingested food. Complex interactions between dietary factors and microorganisms are known to dictate the beneficial or detrimental effects on the host health [8]. Prominently, recent studies have highlighted the importance of gut-derived signals $[9,10]$ and the entity of the GM in the pathogenesis of NASH and NAFLD. In the GM of NAFLD patients, for instance, over-represented Gammaproteobacteria [11,12] and the genera Lactobacillus, Dorea, Robinsoniella and Roseburia [13] have been found compared to healthy controls. Controversially, either low abundance [14] or high abundance [15] of the phylum Bacteroidetes has been detected in NASH patients. Another study reported enriched Fusobacteria, Lachnospiraceae, Enterobacteriaceae, Erysipelotrichaceae and Streptococcaceae in NAFLD patients [16]. These studies indicate that there is no single microbial taxon always positively or negatively associated with liver fat, which, however, may be influenced by the age, gender and geographic location of the study cohorts that knowingly affect the composition of the GM [17-20].

The involvement of the GM in NAFLD has led to the evaluation of possible therapies that either use health-beneficial microbes or target the GM of the host. We have shown that intragastric administration of Faecalibacterium prausnitzii, a commensal member of the GM with known anti-inflammatory properties [21,22], prevented NAFLD in mice [23]. The rationale for investigating this bacterium in the mice model stemmed from our human study, which showed a reverse association of F. prausnitzii abundance with hepatic fat content [24]. Thus, we hypothesized that its low abundance might partly contribute to the diseased phenotype. Our study was in agreement with another study that detected under-represented F. prausnitzii in NASH patients [25].

Our previous findings thus tentatively suggested that NAFLD might be partly relieved with F. prausnitzii. Unfortunately, not all potentially "therapeutic" bacteria are accepted for human use. Therefore, we searched for alternative, nutrition-based tools to increase the natural abundance of F. prausnitzii to treat NAFLD. Such effective tools are known to be probiotics and prebiotics, for instance [26]. A probiotic refers to a live microorganism that positively affects the health of the host, while a prebiotic is defined as a selectively fermented dietary component that cannot be digested as such but serves as food for the GM. A prebiotic thereby causes specific changes in the composition and/or functions of the GM, conferring beneficial effects upon the health of the host. Carbohydrates, such as dietary fiber, are potential prebiotics. These include xylo-oligosaccharides (XOS), fructo-oligosaccharides (FOS), galacto-oligosaccharides (GOS), isomalto-oligosaccharides (IMO), transgalacto-oligosaccharides (TOS) and soybean oligosaccharides (SBOS) [26]. Previously, a $2.8 \mathrm{~g}$ daily dose of XOS, isolated from corncobs, was shown to induce an increase in the abundance of Faecalibacterium species in a group of seven healthy humans [27]. On the contrary, two in vitro studies failed to show any stimulatory effect of XOS on F. prausnitzii growth $[28,29]$. Moreover, a human study did not show effects of XOS on F. prausnitzii but a slightly improved glucose tolerance was achieved in pre-diabetic subjects when they consumed $2 \mathrm{~g}$ of XOS daily, for eight weeks [30].

In the present study, we first tested in vitro whether F. prausnitzii can utilize XOS and how $0.5 \%$ XOS affects the growth of $F$. prausnitzii, and we then studied the effects of a prebiotic dose of XOS $(0.12 \%)$ in vivo. In rats, NAFLD was induced with a high-fat diet (HFD), with or without XOS supplementation. In addition, the effects of XOS (or not) were studied in healthy controls, i.e., in normal, low-fat diet (LFD) fed counterparts that did not generate NAFLD. 


\section{Materials and Methods}

\subsection{In Vitro Cultivations of Faecalibacterium prausnitzii}

In vitro cultivations of F. prausnitzii were done in fastidious anaerobe broth (FAB) supplemented with short-chain fatty acids (SCFA) in an anaerobic workstation (WhitleyA35, Don Whitley Scientific, West Yorkshire, UK). The effects of XOS were studied on two commercially available F. prausnitzii strains, American type of culture collections (ATCC) ${ }^{\circledR}-27766^{\mathrm{TM}}$ (Manassas, VA, USA) and DSM A2-165 (Deutsche Sammlung von Mikroorganismen und Zellkulturen GmbH (DSM), Braunschweig, Germany), with three replicates for both. Ten ml cultures of FAB+SCFA media were supplemented or not with XOS as $0.5 \%(w / v \%)$ as recommended by the scientific advisors of the manufacturer. XOS was isolated from corncobs (Zea mays subsp. mays) by hydrolyzing enzymatically. It was donated by the manufacturer, Shandong Longlive Biotechnology LTD, China (95\% pure, CAS \#87099-0). The growth of F. prausnitzii was determined by measuring changes in the optical density at $620 \mathrm{~nm}$ with a MultiskanFC photometer (Thermo Fisher Scientific, Waltham, MA, USA) after 19, 20.5, 22 and $23.33 \mathrm{~h}$ of cultivations.

\subsection{Animals}

Approval for the animal experiment was received from the National Animal Experiment Board of Southern Finland (ESAVI/8805/4.10.07/2017), and the study was performed in accordance with the Guidelines of the European Community Council directives 2010/63/EU and the European Convention for Protection of Vertebrate Animals used for Experimental and other Scientific Purposes (Council of Europe No123, Strasbourg 1985). In the experiment, the Animal Research: Reporting of In Vivo Experiments (ARRIVE) guidelines were followed. Male Wistar rats, 10-12 weeks of age, were purchased from Charles River, Europe. Wistars were chosen because our pilot studies indicated that they harbor F. prausnitzii in their GM while Sprague Dawley, for instance, do not (data not shown). Upon arrival, the rats were allowed to habituate to the environment for two weeks. During the whole experiment, the rats were single-housed. The rats were divided randomly into four dietary treatment groups ( $n=10$ /group): (1) high-fat diet (HFD, with 60\% of energy from fat), (2) HFD supplemented with XOS (0.12\%, HFD+XOS), (3) control = low-fat diet (LFD, with $10 \%$ of energy from fat), and (4) LFD supplemented with XOS $(0.12 \%, \mathrm{LFD}+\mathrm{XOS})$. It is of note that the control feed contained a standard amount of fat available in the rodent feed, but for clarity and to distinguish it from the high-fat group, it is termed LFD. In humans, a daily dose of $2.8 \mathrm{~g}$, i.e., $0.035 \mathrm{~g} / \mathrm{kg}$, of XOS yielded induction of F. prausnitzii abundance [27] and therefore we targeted a similar dose level in vivo. The average dose of XOS for the rats in our study was $0.05 \mathrm{~g} / \mathrm{kg}$. XOS was isolated as described above and donated by Shandong Longlive Biotechnology (95\% pure, CAS \#87099-0). All irradiated diets were purchased as custom-made pellets from Labdiet/Testdiet, UK. The animals had food and water ad libitum and were maintained in a 12/12 h light/dark cycle in an enriched environment at animal facilities of the University of Jyväskylä. At the beginning of the 12-week diet intervention, all rats were 15 weeks of age.

\subsection{Indirect Metabolic Measurements}

The indirect measures of metabolism were analyzed from respiratory gases with oxygen and $\mathrm{CO}_{2}$ as well as with an analyzer for the capacitive water vapor partial pressure (Promethion ${ }^{\circledR} \mathrm{GA} 3$, Sable Systems, Las Vegas, NV, USA). The air flow was controlled and measured by two multi-channel mass flow generators (FR8, Sable Systems). The incurrent flow rate was set at $3500 \mathrm{~mL} / \mathrm{min}$. The data acquisition was synchronized by MetaScreen ${ }^{\circledR}$ and the raw data were processed with ExpeData $^{\circledR}$ software (Sable Systems). The ExpeData carries out all data transformation, calculating the respiratory quotient (RQ) as the ratio of $\mathrm{CO}_{2}$ production over $\mathrm{O}_{2}$ consumption and energy expenditure by utilizing the equation of Weir: $\mathrm{Kcal} / \mathrm{h}=60 \times\left(0.003941 \times \mathrm{VO}_{2}+0.001106 \times \mathrm{VCO}_{2}\right)[31]$. 


\subsection{Measurement of Weight, Body Composition and Food Intake}

During the study, the animals were weighed once a week always at the same time of day on an electronic scale. The food intake was measured once a week by weighing the consumed feed over $24 \mathrm{~h}$. The body composition was determined with dual energy x-ray absorptiometry (DXA, Prodigy; GE Lunar Corp., Madison, WI, USA) under isoflurane anesthesia before and after the diet intervention.

\subsection{Necropsy, Blood Analyses, Tissue Collection and Histology}

After the 12-week diet intervention, the non-fasted rats were anesthetized with a mixture of air and $\mathrm{CO}_{2}$ and euthanized by drawing the blood by cardiac puncture. KONELAB 20XTi analyzer (Diagnostic Products Corporation, Los Angeles, CA, USA) was used to determine the serum levels of glucose, triglycerides, free fatty acids, glycerol, cholesterol, LDL (low-density lipoprotein) cholesterol, HDL (high-density lipoprotein) cholesterol, aspartate aminotransferase (AST) and alanine aminotransferase (ALT). Serum insulin was measured with IMMULITE analyzer (Siemens healthineers, Erlangen, Germany). Serum cytokines were analyzed with 9-plex cytokine ELISA kit (\#110449RT), Quansys and Q-View software (Quansys Biosciences, Logan, UT, USA), as specified in the instructions of the manufacturer. The detection limits for the cytokines were as follows: interleukin (IL)-1a, $8.55 \mathrm{pg} / \mathrm{mL} ; \mathrm{IL}-1 \beta, 3.58 \mathrm{pg} / \mathrm{mL}$; IL-2, $2.74 \mathrm{pg} / \mathrm{mL}$; IL-4, $0.45 \mathrm{pg} / \mathrm{mL}$; IL-6, $1.4 \mathrm{pg} / \mathrm{mL} ; \mathrm{IL}-10,0.26 \mathrm{pg} / \mathrm{mL}$; IL-12, $0.41 \mathrm{pg} / \mathrm{mL}$; interferon (IFN)- $\gamma, 33.71 \mathrm{pg} / \mathrm{mL}$ and tumor necrosis factor (TNF)- $\alpha, 2.72 \mathrm{pg} / \mathrm{mL}$.

The medial lobe of the liver was harvested and, after excising the samples for the mitochondrial respiration analysis and histology, the rest of the medial lobe was immersed in liquid nitrogen and stored at $-80^{\circ} \mathrm{C}$. For the subsequent analyses of enzyme activity, fat content and mRNA expression, the frozen medial lobe was pulverized in liquid nitrogen. For histology, a piece of the liver was snap-frozen in cooled isopentane $\left(-150{ }^{\circ} \mathrm{C}\right)$ and stored at $-80^{\circ} \mathrm{C}$. Neutral lipids were visualized from $10 \mu \mathrm{m}$ cryosections with Oil Red O staining. Paraformalin-fixed sections were rinsed with $\mathrm{H}_{2} \mathrm{O}$, stained for 15 min with freshly prepared Oil Red O solution (Merck, Kenilworth, NJ, USA) and rinsed with $60 \%$ isopropanol to avoid over-staining. The sections were counterstained with Mayer's hematoxylin and scanned with NanoZoomer microscope (Hamamatsu, Japan). The amount of hepatic fat was scored by two blinded experimenters.

To estimate the amount of liver fibrosis, $10 \mu \mathrm{m}$ paraformaldehyde-fixed cryosections were stained with Sirius Red. The sections were first stained with Weigert's hematoxylin (Sigma-Aldrich, St Louis, MO, USA), then washed with tap water and stained in Sirius Red (Sigma-Aldrich)-picric acid (Sigma-Aldrich) solution. Finally, the sections were immersed twice in acidified water and cover-slipped with Depex following dehydration and clearing in ethanol and xylene. Then, the sections were scanned with NanoZoomer and the amount of fibrosis was scored from the scanned images by two blinded experimenters. The scoring was based on Ishak grade [32]. Briefly, 0 is no fibrosis; 1 is fibrous expansion of some portal areas and short fibrous septa; 2 is fibrous expansion of most portal areas and short fibrous septa; 3 is fibrous expansion of most portal areas and occasional portal to portal bridging; 4 is fibrous expansion of portal areas and marked portal to portal as well as portal to central bridging; 5 is marked bridging and occasional nodules, and 6 is probable or definite cirrhosis.

For the histology of the gut, $10 \mathrm{~mm}$ of proximal colon was cut right after the cecum, the colon contents were collected (see below) and the rest of the feces was washed out with phosphate-buffered saline (PBS). The collected tissue was fixed with $4 \%$ paraformaldehyde for $48 \mathrm{~h}$, washed twice with PBS and then stored in $70 \%$ ethanol at $4{ }^{\circ} \mathrm{C}$. The histopathological scoring of the proximal colon was done by a veterinarian and expert toxicopathologist (author J.L.). For the assessment, the formalin-fixed proximal colon samples of approximately $1 \mathrm{~cm}$ of length were trimmed into 4-6 pieces and embedded in paraffin transversally. The paraffin blocks were cut at $3 \mu \mathrm{m}$, resulting in 4-6 transversal sections per colon sample. The sections were stained with hematoxylin and eosin. The microscopic findings were classified with standard pathological nomenclature and severities of inflammatory activity, mucosal atrophy and crypt hyperplasia were graded on a suitable scale (generally 1 to 5 as minimal, mild, moderate, marked or severe). The grades of severity for the microscopic findings were subjective; 
minimal was the least extent discernible and severe was the greatest extent possible. The grading was based on both the severity and extent (focal, extensive and diffuse) of the affection. The crypt hyperplasia was not assessable in some cases. A detailed description of the scoring is provided in the Supplementary Materials.

To visualize intestinal tight junctions, the $3 \mu \mathrm{m}$ sections were deparaffinized and boiled in $0.01 \mathrm{M}$ sodium citrate ( $\mathrm{pH}$ 6.0) for antigen retrieval and blocked with $10 \%$ goat serum. The intestinal tight junctions were stained with anti-tight junction protein-1 antibody (Tjp1, Novus Biologicals, Littleton, CO, USA) and visualized by labeling with anti-rabbit 647 Alexa Fluor (Invitrogen, Carlsbad, CA, USA). The nuclei were made visible with DNA-stain 4',6-diamidino-2-phenylindole (DAPI, 1:2000), and then the sections were cover-slipped with Mowiol-mounting media. The labeled sections were imaged with a confocal microscope (Carl Zeiss LSM 700) and the signal intensities were counted with Image J. From each rat, four randomly selected areas were imaged, with four tiles per area $(1184 \mu \mathrm{m} \times 1184 \mu \mathrm{m})$. The fluorescence intensity was normalized to the intensity of DAPI.

\subsection{Collection of Gut Contents, DNA Extraction and Real-Time Quantitative PCR}

The contents of the proximal colon and cecum were collected at necropsy, snap-frozen in liquid nitrogen and stored at $-80{ }^{\circ} \mathrm{C}$. The total DNA was extracted from $100 \mathrm{mg}$ of the colon and cecum contents with Stool Extraction Kit and semi-automated GenoXtract (Hain Lifescience $\mathrm{GmbH}$, Nehren, Germany), accompanied by bead-beating in $1.4 \mathrm{~mm}$ ceramic bead tubes to ensure better homogenization.

Real-time quantitative PCR (qPCR) was done using DNA extracted from the cecum and F. prausnitzii $16 \mathrm{~S}$ rRNA-targeted primers, as has been described previously [33]. First, in the traditional PCR, pure cultures of F. prausnitzii and F. prausnitzii 16S rRNA-targeted primers were used. The PCR program was as follows: pre-incubation at $95^{\circ} \mathrm{C}$ for $10 \mathrm{~min}, 40$ cycles at $95{ }^{\circ} \mathrm{C}$ for $30 \mathrm{~s}, 60{ }^{\circ} \mathrm{C}$ for $1 \mathrm{~min}$ and $72{ }^{\circ} \mathrm{C}$ for $30 \mathrm{~s}$, followed by a final extension at $72{ }^{\circ} \mathrm{C}$ for $8 \mathrm{~min}$ (Veriti 96 Well Thermal Cycler, Applied Biosystems, Foster City, CA, USA). The expected size of the PCR product was 140 base pairs. After obtaining and purifying the PCR products, a dilution series for the qPCR standard curve was made by pooling the positive PCR products obtained in the traditional PCR. The concentration of the pooled DNA fragments was measured with NanoDropND-1000 spectrophotometer (ND, Nanodrop Technologies Inc., Wilmington, DE, USA). Taking into account that the molecular weight of one DNA base pair is $660 \mathrm{~g} / \mathrm{mol}$ and that the size of the PCR product was 140 base pairs, the concentration of DNA fragments in the pool could be calculated based on the DNA concentration: DNA concentration $(\mathrm{ng} / \mu \mathrm{L}) /$ molecular weight of one PCR fragment $(\mathrm{g} / \mathrm{mol})=$ fragment concentration. Then, by multiplying the fragment concentration $(\mathrm{mol} / \mu \mathrm{L})$ with Avogadro's number $(6.0221415 \times 1023)$, the number of fragments per one $\mu \mathrm{L}$ was obtained. Then, a dilution series from $10^{10}$ to $10^{1}$ was done and used as a standard curve in the subsequent qPCR. The qPCR results are shown as F. prausnitzii gene copy number/gram of cecal content used to extract the DNA. However, it should be noted that the gene copy number is not likely to be representative of the absolute bacterial cell numbers of F. prausnitzii in the samples, because it is not known how many gene copies of 16S rRNA each single F. prausnitzii cell has.

\section{7. $16 S$ rRNA Gene Sequencing and Processing of the Sequence Data}

For the microbial community analysis, rRNA gene was amplified using primers 515F-Y (GTGYCAGCMGCCGCGGTAA) and 806R (GGACTACHVGGGTWTCTAAT) that target the V4 region of the subunit ribosomal RNA (SSU rRNA) gene. In the first PCR, the reaction consisted of 1xMaxima ${ }^{\mathrm{TM}}$ SYBR Green $\mathrm{qPCR}$ Master Mix (Thermo Fisher Scientific, Waltham, MA, USA), $0.5 \mu \mathrm{M}$ of primer and $20 \mathrm{ng}$ of DNA template. The PCR program was as follows: $10 \mathrm{~min}$ initial denaturation at $95{ }^{\circ} \mathrm{C}, 30$ cycles at $94{ }^{\circ} \mathrm{C} 30 \mathrm{~s},+52{ }^{\circ} \mathrm{C} 60 \mathrm{~s}$ and $72{ }^{\circ} \mathrm{C} 60 \mathrm{~s}$, followed by a final extension at $72{ }^{\circ} \mathrm{C}$ for $5 \mathrm{~min}$ (C1000 ThermalCycler, Bio-Rad Laboratories, Hercules, CA, USA). After this, Ion Torrent PGM sequencing adapters and barcodes were added to the ends of the PCR product by using one $\mu \mathrm{L}$ of the PCR product as a template in the second PCR. In this second PCR, 10 cycles were performed 
using linker and fusion primers $(0.05 \mu \mathrm{M}$ of M13_515F-Y, $0.5 \mu \mathrm{M}$ of IonA_IonXpressBarcode_M13 and P1_806R), the other conditions being identical to the first PCR. The sequencing was done using Ion Torrent PGM (Thermo Fisher Scientific). The PCR products were purified with AMPure XP (Beckman Coulter, Brea, CA, USA), quantified with PicoGreen (Quant-iT ${ }^{\mathrm{TM}}$ PicoGreen ${ }^{\mathrm{TM}}$ dsDNA Assay Kit, Thermo Fisher Scientific, Waltham, MA, USA) and pooled in equimolar quantities for sequencing on Ion Torrent PGM using Hi-Q View OT2 Kit for emulsion PCR, Hi-Q View Sequencing Kit for the sequencing reaction and Ion 318 Chip v2 (Thermo Fisher Scientific).

Quality filtering and clustering to the operational taxonomic units (OTUs) at the 97\% similarity level of the 16S rRNA gene sequences was carried out using the CLC Microbial Genomics Package (Qiagen, Hilden, Germany). After preprocessing, the dataset contained 5.0 million reads-on average, $64,544 \pm 15,932$ reads per sample and 12,700 unique OTUs. The rRNA gene sequences were classified using the SILVA SSU Ref database (v132, 99\%). First, the GM diversity was analyzed from the cecal and colon contents, separately. Because no differences in the distribution of evenness between species, i.e., alpha-diversity of the GM, were observed between the two intestinal compartments (see Results, Section 3.2), the sequence data of the cecum and colon samples were pooled for further analyses of the GM composition.

\subsection{Extraction, Identification and Analyses of Cecal Metabolites}

Ice-cold PBS (pH 7.4) was mixed with the cecal content at a ratio of 1:2 and vortexed for 5 min to extract cecal metabolites. The extract was then centrifuged at $15,000 \times g$ for $15 \mathrm{~min}$ at $4{ }^{\circ} \mathrm{C}$. A part of the resultant supernatant was mixed with $10 \%$ Chenomx standard solution ( $5 \mathrm{mM}$ deuterated DSS (DSS-d6) and sodium azide in $\mathrm{D}_{2} \mathrm{O}$ ) and vortexed for $15 \mathrm{~s}$. Then, $180 \mu \mathrm{L}$ of the prepared mix was placed into $3 \mathrm{~mm}$ nuclear magnetic resonance (NMR) tubes. The spectra were recorded using a $600 \mathrm{MHz}$ Bruker AVANCE-III NMR spectrometer, which was equipped with TCI Prodigy CryoProbe (Bruker BioSpin AG, Fällanden, Switzerland).

The recording of the NMR spectra was performed using a $600 \mathrm{MHz}$ Bruker AVANCE-III NMR spectrometer (Bruker BioSpin AG, Fällanden, Switzerland) that was equipped with a TCI Prodigy CryoProbe. A 128-scan Carr-Purcell-Meiboom-Gill sequence (CPMG) pulse was applied to acquire the spectra consisting of $128 \mathrm{k}$ data points at a spectral width of $10 \mathrm{kHz}$, at $25{ }^{\circ} \mathrm{C}$, with an acquisition time of $6.82 \mathrm{~s}$. To acquire the J-resolved (JRES) spectra, the parameters that we used were 16 scans, $1 \mathrm{k}$ data points, 128 increments, $2 \mathrm{~s}$ relaxation delay and a spectral width of $16 \mathrm{ppm}$ in dimensions. The heteronuclear single quantum coherence (HSQC) spectra were acquired using 32 scans, $2 \mathrm{k}$ data points, 128 increments, 2 s relaxation delay and spectral width of $16 \mathrm{ppm}$ and $165 \mathrm{ppm}$ in the proton and carbon dimensions, respectively.

\subsection{Processing of the Cecal Metabolite Data and Multivariate Data Analysis}

The metabolites were identified and quantified with the aid of Chenomx NMR Suite 8.3 Professional (Chenomx Inc., Edmonton, Alberta, Canada), Human Metabolome Database (HMDB, http://www. hmdb.ca), and Biological Magnetic Resonance data Bank (BMRB, http://www.bmrb.wisc.edu). Two-dimensional JRES and HSQC spectra were used in confirming the identities of the metabolites.

The ${ }^{1} \mathrm{H}$ NMR spectra were manually phased, baseline and shim-corrected and referenced to trimethylsilylpropanoic acid (TSP) at 0.00 ppm using Chenomx NMR Suite. The misaligned regions of the spectral set were corrected using icoshift. After removal of the residual water (4.68-4.88) region, the spectra were subjected to total area normalization and then reduced to variable sized bins of width ranging from 0.018 to $0.04 \mathrm{ppm}$. This resulted in a dataset comprising 40 observations and 157 variables. The pareto-scaled dataset was then subjected to unsupervised (PCA) and supervised multivariate analyses using (PLS-DA and OPLS-DA) using SIMCA-P 14.1 (Umetrics, Sartorius Stedim Biotech, Umeå, Sweden). The validation of the supervised multivariate models was achieved by 100 permutation test, estimation of explained variation (R2Y (cum)), predictive ability (Q2Y (cum)) and CV-ANOVA values. 


\subsection{Measurement of the Liver Fat Content and 3-Hydroxyacyl-CoA Dehydrogenase Activity}

To extract the total lipids, a pulverized sample of the medial lobe of the liver was analyzed with KONELAB 20XTi, as described previously by us [34]. To analyze the activity of 3-hydroxyacyl-Coenzyme A (CoA) dehydrogenase 8 ( $\beta$-HAD), $20 \mathrm{mg}$ of pulverized liver was homogenized with cold lysis buffer $(10 \mathrm{mM}$ Tris- $\mathrm{HCl}, 150 \mathrm{mM} \mathrm{NaCl}, 2 \mathrm{mM}$ ethylenediaminetetraacetic acid (EDTA), 1\% Triton X-100, 10\% glycerol and $1 \mathrm{mM}$ dithiotreitol (DTT)) that contained protease and phosphatase inhibitors (Sigma Aldrich, St Louis, MO, USA) using TissueLyzer (Qiagen, Valencia, CA, USA). After separating the insoluble material by centrifugation at $12,000 \times g$, $\beta-H A D$ activity was determined from the supernatant with KONELAB 20XTi. For the measurement,

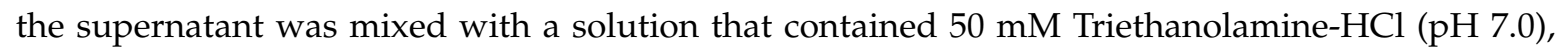
4 mM EDTA, 0.04 mM NADH and 0.015 mM S-Acetoacyl CoA.

\subsection{Measurement of Hepatic Mitochondrial Functions with High-Resolution Respirometry to Analyze the} Rate of Hepatic Glucose Metabolism

A freshly collected sample of the medial lobe of the liver (15-20 mg) was homogenized in 0.5 mL Mir05-medium with PBI-Schredder high-resolution respirometry set (Oroboros instruments, Innsbruck, Austria). Shredding was done for $10 \mathrm{~s}$ 1-level $+5 \mathrm{~s}$ 2-level. The wash of the shredding pipe was performed by applying three times $0.5 \mathrm{~mL}$ of Mir05-medium. Medium was collected into a clean tube with the homogenate, and the final volume was set to $5 \mathrm{~mL}$ with Mir05-buffer. An aliquot of $0.8 \mathrm{~mL}$ of the homogenate and $1.5 \mathrm{~mL}$ of Mir05-medium was transferred to the Oroboros O2k-Respirometer (Oroboros instruments). DatLab software (Oroboros instruments) was used to record oxygen concentration $(\mu \mathrm{M})$ and oxygen flux per tissue wet mass $\left(\mathrm{pmol} \mathrm{O} \bullet \bullet \mathrm{s}^{-1} \bullet \mathrm{mg}^{-1}\right)$. Oroboros program was performed as follows: (1) Pyruvate, malate and glutamate (5 mM, $2 \mathrm{mM}, 10 \mathrm{mM})$ were used as initial substrates but without adenosine diphosphate (ADP) leak respiration being measured. (2) The addition of ADP and $\mathrm{Mg}^{2+}(4 \mathrm{mM}, 2.4 \mathrm{mM})$ started oxidative phosphorylation through mitochondrial complex I (CI). (3) Cytochrome c $(10 \mu \mathrm{M})$ was added in order to monitor unwanted mitochondrial degradation. (4) Succinate $(10 \mathrm{mM})$ was added as a complex II substrate (CI+II). (5) The maximal capacity of the electron transport system was measured by adding carbonyl cyanide m-chlorophenyl hydrazone (CCCP) (0.5-2.5 $\mu \mathrm{M}$; until max $\mathrm{O}_{2}$ consumption was reached). (6) The complex I was inhibited with rotenone $(0.5 \mu \mathrm{M})$ and (7), complex III with antimycin A $(2.5 \mu \mathrm{M})$ and finally only the residual oxygen consumption (ROX) was left. ROX was subtracted from all other oxygen flux values and all values were expressed as normalized to the wet tissue mass.

\subsection{2. qPCR of Liver and Adipose Tissues}

The total RNA from the pulverized liver as well as mesenteric and epididymal adipose tissues was extracted using Tri reagent (Thermo Fisher Scientific) according to the protocol provided by the supplier. The RNA was reverse transcribed into cDNA using High Capacity cDNA synthesis kit as specified in the instructions of the manufacturer (Applied Biosystems). The real-time PCR analysis was done according to the Minimum Information for Publication of Quantitative Real-Time PCR Experiments (MIQE) guidelines using in-house designed primers (from Invitrogen), iQ SYBR Supermix and CFX96 ${ }^{\mathrm{TM}}$ Real-time PCR Detection System (Bio-Rad Laboratories, Hercules, CA, USA). The primer sequences were as follows: stearoyl conenzyme saturase 1 (SCD1) forward 5'CCTCATCATTGCCAACACCAT3' and reverse 5'AGCCAACCCACGTGAGAGAA3'; diacylglycerol O-acyltransferase 2 (DGAT2) forward 5'GGGTCCAGAAGAAGTTCCAGAAG3' and reverse 5'CCAGGTGTCAGAGGAGAAGAG3'; IL1beta forward 5'CACAAAAATGCCTCGTGC3' and reverse 5'TGCTGATGTACCAGTTGGG3'; leptin forward 5' AGCAGTGCCTATCCAGAAGT3' and reverse 5' ${ }^{\prime}$ TTCTCCAGGTCATTGGCTAT3'; adiponectin forward 5' AATCCTGCCAGTCATGAAG3' and reverse 5'CATCTCCTGGGTCACCCTTA3'. 


\subsection{Statistical Analyses}

The statistical analyses, except for the GM and their metabolites, were performed with IBM SPSS Statistics v24 for Windows (SPSS, Chicago, IL, USA). The main effects of the diet and XOS were determined using a general linear model or mixed model analysis. The group differences were analyzed with ANOVA. Kruskal-Wallis or median test was used if the data were not normally distributed according to the Shapiro-Wilk test in SPSS. For the repeated measures, we used linear mixed model. Type III tests of fixed effects with Sidak's adjustment for multiple comparisons were used. Cohen's $d$ was used to estimate differences between the groups in F. prausnitzii abundance because of the high inter-individual variation. The associations between the variables were studied with Spearman's correlation coefficient.

The alpha-diversity of the GM was quantified with Shannon index using the microbiome $\mathrm{R} /$ Bioconductor package. The analysis of beta-diversity was based on Bray-Curtis distance and PERMANOVA [35] for significance testing (adonis function in the vegan $\mathrm{R}$ package). The taxonomic groups were compared based on DESeq2 [36] using the phyloseq R/Bioconductor package, including Benjamini-Hochberg correction for multiple testing. The statistical significance was set at $p<0.05$ after the multiple testing corrections.

The univariate analysis of the concentrations of cecal metabolites was performed using Graph Pad Prism 8.0 (GraphPad Software, San Diego, CA, USA). The normal distribution of the data was tested with the Shapiro-Wilk test. The differences between the groups were assessed with the parametric one-way ANOVA for normally distributed variables and with Tukey's multiple comparison test or non-parametric Kruskal-Wallis test for the non-normally distributed variables with Dunn's multiple comparisons. The statistical significance was set at $p<0.05$.

\section{Results}

\subsection{XOS Increased the Growth of Faecalibacterium Prausnitzii and Concomitantly Decreased Hepatic Fat Content Due to Enhanced Fat and Glucose Metabolism}

We first tested in vitro whether F. prausnitzii can utilize XOS and how XOS affects its growth. Compared to the control treatment, XOS increased the growth of $F$. prausnitzii in vitro $(\mathrm{F}(1,16.9)=64.9$, $p<0.001)$. The effects of time $(\mathrm{F}(3,25.6)=5.4, p=0.005)$ and cell line $(\mathrm{F}(1,16.9)=59.6, p<0.001)$ were also significant. Thus, the difference between the control and XOS treatment was significant at each time point (after 19, 22, 23.3 h of stimulation) in the DSM A5-165 strain $(p<0.05)$ but not in the ATCC-27766 strain (Figure 1a). In rats, after 12 weeks of dietary XOS supplementation, F. prausnitzii abundance was increased compared to the HFD (Cohen's $d=0.2)$ and LFD (Cohen's d $=0.2$ ) without XOS (Figure 1b). Real-time quantitative PCR was used to analyze F. prausnitzii abundance in rats, because unfortunately, the primers used in the 16S rRNA gene sequencing did not catch F. prausnitzii.

As expected, the biochemical measurement of the hepatic triglyceride content showed that the HFD increased triglycerides $(F(1,6.0)=46.4, p<0.001)$. XOS decreased triglycerides on the HFD $(F(1,0.8)=6.5, p=0.017)$, while when supplemented with LFD, XOS increased hepatic triglycerides (Figure 2a). A similar interactive effect of XOS was found on the total hepatic cholesterol $(\mathrm{F}(1,0.2)=15.2, p=0.001)$, although in group comparisons only the LFD+XOS had significantly higher hepatic cholesterol content than the LFD ( $p=0.015$, Figure 2a). To confirm the findings on the content of hepatic triglycerides, the medial lobe of the liver was analyzed also histologically. Oil Red O staining of the frozen liver sections showed that XOS supplementation decreased hepatic neutral lipids compared to the HFD in rats (Figure 2b). Based on the Sirius Red staining, there was no pronounced progression to hepatic fibrosis in any of the diet groups or differences in the fibrosis score between the groups. Yet, there was, to some extent, fibrous expansion in portal areas and short fibrous septa in all groups, which could be due to having feed ad libitum. Examples of typical stainings are shown in Supplementary Figure S1. 


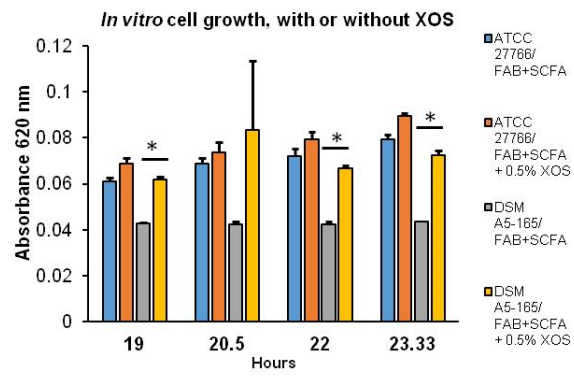

(a)

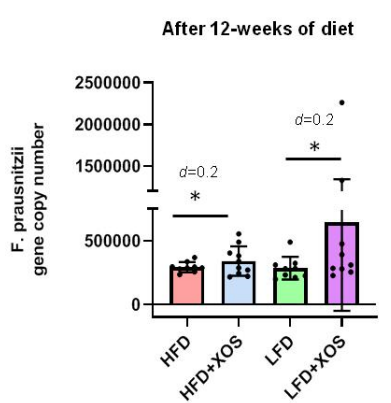

(b)

Figure 1. Xylo-oligosaccharides (XOS) increased the growth of $F$. prausnitzii in vitro and in vivo. (a) In vitro stimulation of F. prausnitzii growth with XOS. Cultivations of F. prausnitzii were done in fastidious anaerobe broth (FAB) supplemented with short-chain fatty acids (SCFA) in an anaerobic workstation. Ten mL cultures of commercially available F. prausnitzii strains American type of culture collections (ATCC) ${ }^{\circledR}-27766^{\mathrm{TM}}$ and Deutsche Sammlung von Mikroorganismen und Zellkulturen GmbH (DSM) A2-165 were supplemented or not with XOS as $0.5 \%(w / v \%)$. The growth of F. prausnitzii was determined by following changes in the optical density at $620 \mathrm{~nm} . n=4$ replicates/treatments. * indicates statistically significant difference between the groups; (b) Quantitative real-time PCR of F. prausnitzii using DNA extracted from the rat cecum after 12 weeks of diet. The results are shown as gene copy number divided per gram of cecum content used to extract the total bacterial DNA. Cohen's $d$ was used to estimate the differences between the groups. $n=8-10 /$ diet group. The different data points are shown with black dots. HFD = high-fat diet. LFD = low-fat diet. * indicates statistically significant difference between the groups.
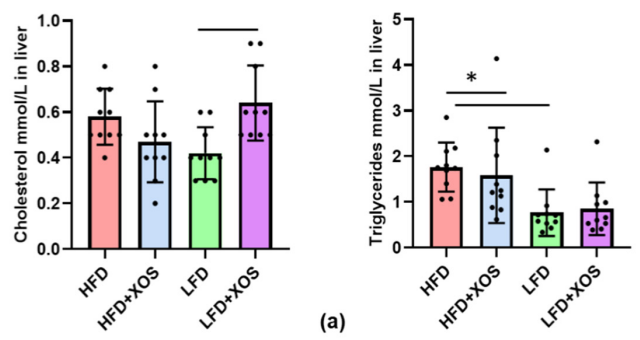

HFD

$\mathrm{HFD}+\mathrm{XOS}$

LFD

LFD + XOS
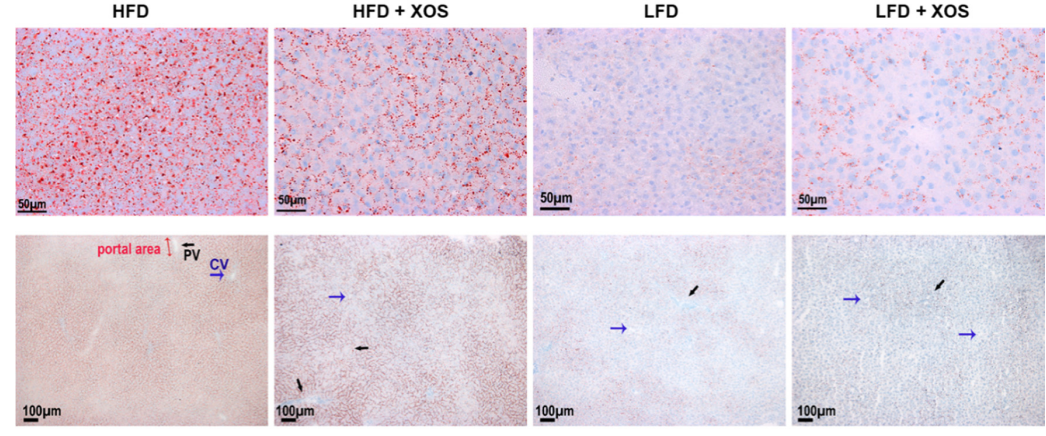

(b)

Figure 2. XOS decreased hepatic fat content in rats fed with HFD. (a) The biochemical analyses of the total hepatic cholesterol and triglyceride content. $n=8-10 /$ diet group. The different data points are shown with black dots. * indicates statistically significant difference between the groups; (b) The frozen liver sections of the rats stained with Oil Red O. The scale bar in the upper images is $50 \mu \mathrm{M}$ and in the lower images $100 \mu \mathrm{M}$. The histological images were taken with Olympus BX50 microscope and Colorview III camera using 40× (Olympus UPlanFI, NA 0.75) and 10× (Olympus UPlanFI NA 0.3) objectives. Blue arrows indicate central vein, which is abbreviated as $\mathrm{CV}$ in the lower image of the HFD. Black arrows indicate portal vein, which is abbreviated as PV in the lower image of the HFD. 
To assess whether the decreased hepatic fat content was due to increased fat oxidation, we determined the activity of $\beta$-HAD, the rate-limiting enzyme of fatty acid $\beta$-oxidation. XOS increased the hepatic activity of $\beta$-HAD on the $\operatorname{HFD}(\mathrm{F}(1,36)=4.5, p=0.041)$ (Figure 3a).

We then analyzed hepatic glucose metabolism using high-resolution respirometry. In the hepatic mitochondria, the HFD decreased maximal electron transport $(p=0.034)$ (Figure $3 b)$. The HFD also lowered the maximal electron transport capacity theoretically available for the oxidative phosphorylation $(p=0.023)$, and the reserve electron transport capacity beyond the oxidative phosphorylation through complex I ( $p=0.019)$ as well as complexes I and II ( $p=0.013$ ) (Figure 3c). XOS supplementation seemed to ameliorate these effects of the HFD on mitochondrial respiration (Figure 3c). Compared to the HFD, HFD+XOS had increased respiratory capacity available for the production of ATP through the electron flow from complex I ( $p=0.023$, Figure 3c) and improved coupling of electron transport through complex I and oxidative phosphorylation ( $p=0.041$, Figure 3d).

The HFD decreased but XOS had no effects on the hepatic mRNA expression of SCD1 (effect of diet: $\mathrm{F}(1,36)=97.5, p<0.001$ ) and DGAT2 (effect of diet: $\mathrm{F}(1,35)=8.7, p=0.006$ ), which are involved in lipogenesis and the synthesis of triglycerides, respectively (Figure 4).

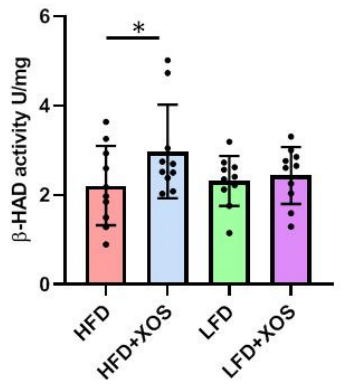

(a)

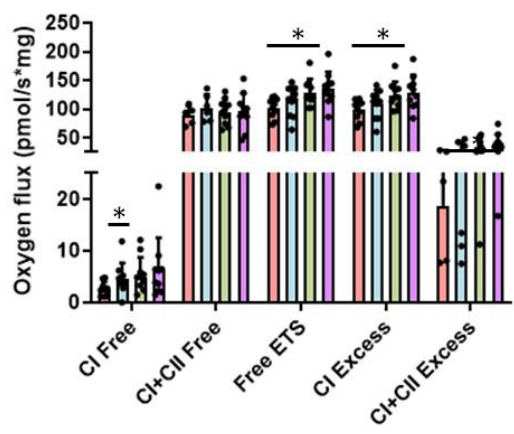

(c)

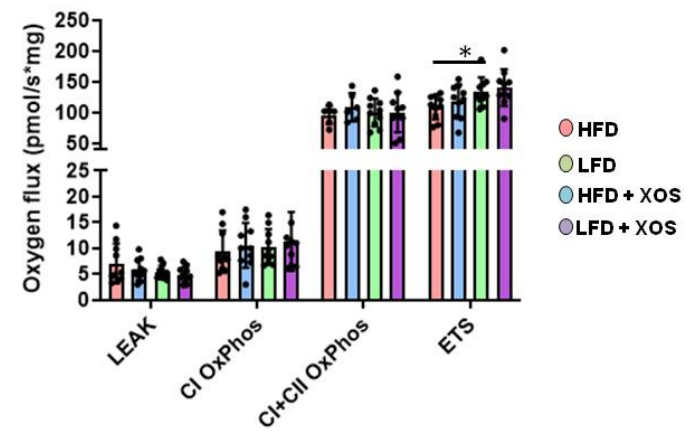

(b)

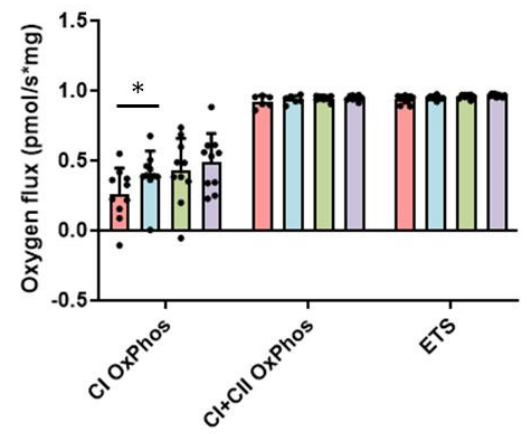

(d)

Figure 3. XOS enhanced the hepatic activity of fatty acid-oxidating beta-hydroxydeacetylase dehydrogenase ( $\beta$-HAD) on the HFD and also increased mitochondrial respiration, reflecting increased glucose metabolism. (a) The biochemically measured activities of $\beta$-HAD in rat livers. $n=8-10 /$ diet group; (b) HFD lowered maximal electron transport (ETS) in liver mitochondria. $n=8-10 /$ diet group; (c) HFD lowered maximal electron transport capacity available for oxidative phosphorylation (Free ETS), reserve electron transport capacity beyond oxidative phosphorylation through complex I (CI Excess) as well as through complexes I and II (C+CII Excess). On the HFD, XOS increased the respiratory capacity available for the production of ATP through the electron flow from complex I (CI Free). $n=8-10$ /diet group; (d) On the HFD, XOS improved coupling of electron transport through the complex I and oxidative phosphorylation (CI OxPhos, coupling efficiency). $n=8-10 /$ diet group. In all graphs, the different data points are shown with black dots. * indicates statistically significant difference between the groups in all graphs. 

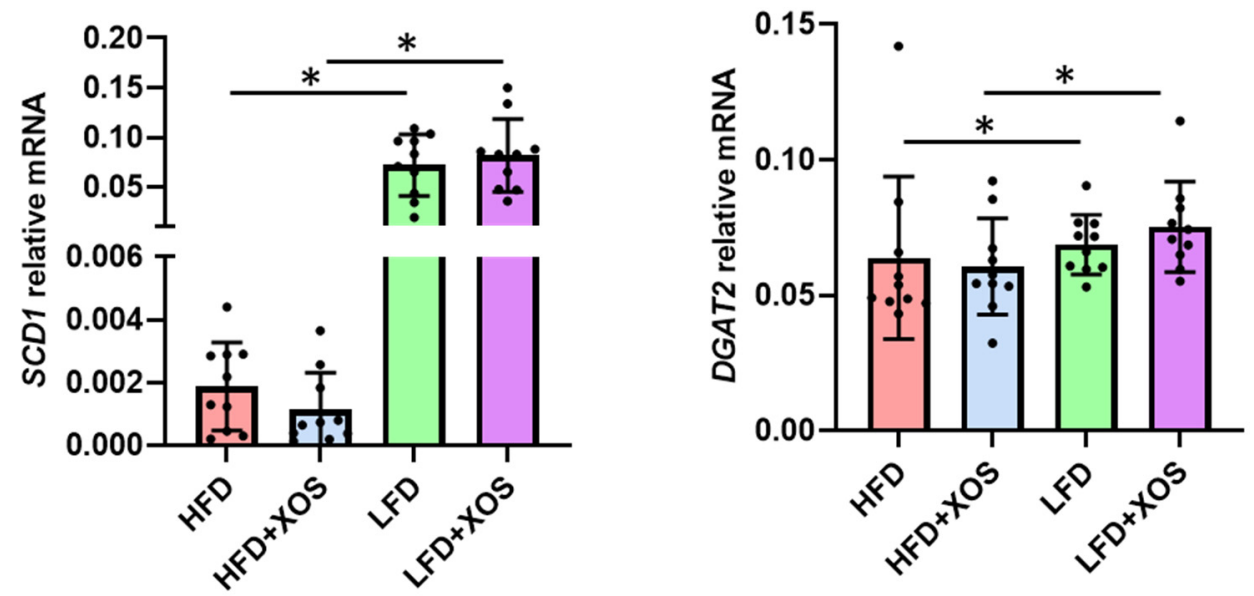

Figure 4. XOS did not affect the hepatic mRNA expression of stearoyl coenzyme desaturase 1 (SCD1) or Diacylglycerol O-acyltransferase 2 (DGAT2). $n=8-10 /$ diet group. The different data points are shown with black dots. ${ }^{*}$ indicates statistically significant difference between the groups.

3.2. The Diets Did Not Affect the Diversity of the Gut Microbiota, but Minor Differences Were Found in the Relative Abundances of Three Microbial Genera

Despite the over 12,000 OTUs, the proximal colon and cecum of the rats were dominated by a few phyla and genera. Bacteroidetes (51.6\% of all sequences) and Firmicutes (39.7\%) were the dominating bacterial phyla, followed by Verrucomicrobia (3.6\%) and Proteobacteria (2.3\%). The families Tannerellaceae (19.1\%, only genus Parabacteroides), Rikennellaceae (14.6\%, mostly genus Alistipes) and Muribaculaceae (10.0\%, several genera) explained the dominance of Bacteroidetes, and the families Ruminococcaceae (16.4\%) and Lachnospiraceae (13.3\%) were the dominating sequences belonging to Firmicutes. The hierarchical clustering analysis suggested two main clusters among the rat groups based on their GM profiles. The dietary fat explained the clusters regardless of XOS supplementation (data not shown). However, the diets did not affect the alpha-diversity (Figure 5a) or the beta-diversity (Figure 5b) of the colon or cecum GM. The GM composition did not differ between the groups at phylum (Figure 6a) or family level, including the Ruminocaccaceae family, to which F. prausnitzii belongs (data not shown). At genus level, Dubosiella and uncultured members of Christensenellaceae were lowest and the Prevotellacaeae NK3B31 group highest in the HFD+XOS ( $p=0.01$ for all, Figure 6b). The GM of the HFD tended to have higher relative abundance of Prevotellacaeae UCG-10 $(p=0.06)$, and LFD groups had higher abundance of Anaerostipes ( $p=0.10)$ (data not shown).

\subsection{XOS Improved HFD-Induced Intestinal Damage and Inflammatory Markers in the Proximal Colon, but Did Not Affect Intestinal Tjp1, Hepatic IL1ß mRNA or Systemic Inflammation}

In the histopathological examination of the proximal colon, an interactive effect of the diet and XOS was found on the surface epithelial injury $(\mathrm{F}(1,35)=35.0, p<0.001)$, Goblet cell hyperplasia $(\mathrm{F}(1,35)=12.6, p=0.001)$, crypt length distortion $(\mathrm{F}(1,35)=31.0, p<0.001)$ and injury score $(\mathrm{F}(1,35)=90.4, p<0.001)$ (Figure 7$)$. XOS diminished the epithelial injury caused by the HFD, while on the LFD, XOS increased the injury $(\mathrm{F}(1,35)=18.9, p<0.001)$ (Figure 7$)$. On the LFD, XOS also increased monocyte aggregates, i.e., leukocyte infiltration to the lamina propria $(F(1,36)=5.8, p=0.021)$ but had no effects on lymphocyte aggregates in lamina propria, i.e., cryptopatches or large granular lymphocytes (Figure 7).

However, despite these important changes in the epithelia and the fact that HFD is known to compromise gut integrity and, conversely, prebiotic nutrients are known to enhance it, the histological analysis of the tight junctions showed no differences between the groups (Figure 8). 

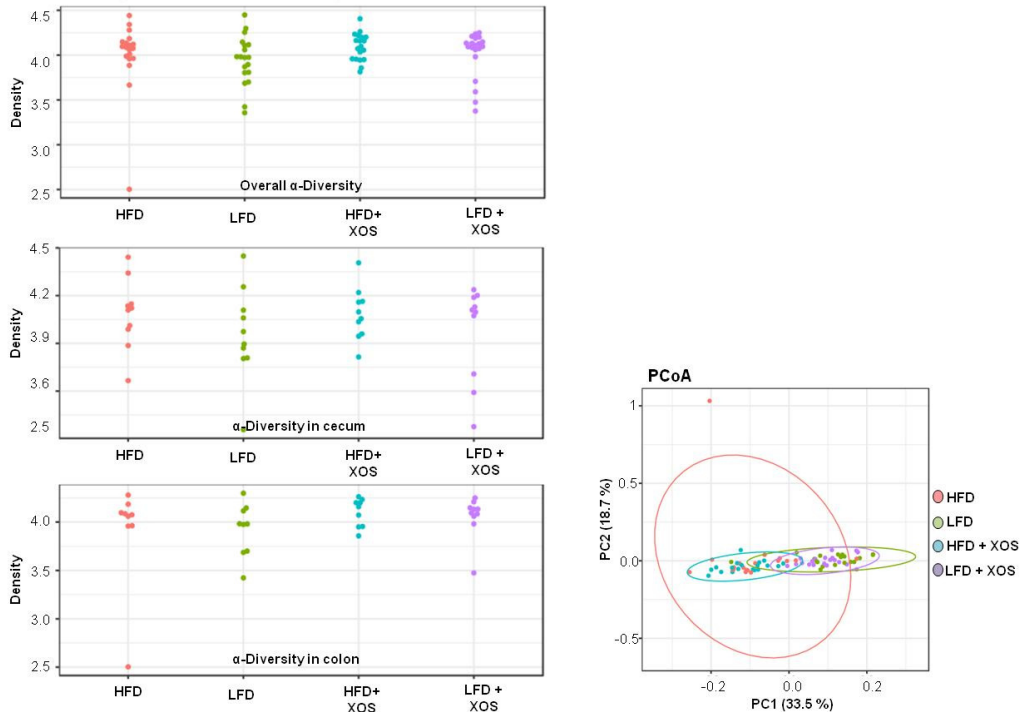

(a)

(b)

Figure 5. No significant associations were observed between the diets and the diversity of the gut microbiota. (a) The overall alpha-diversity (on top), alpha-diversity in colon (at the middle) and cecum (on bottom) of the gut microbiota. $n=10$ /diet group. The alpha-diversity of the gut microbiota was quantified with Shannon index using microbiome R/Bioconductor package; (b) The beta-diversity of the gut microbiota according to the principal component analysis (PCoA). PC1 indicates principal component 1 and PC2 principal component 2. $n=10 /$ diet group. The analysis of beta-diversity was based on Bray-Curtis distance, and PERMANOVA was used for significance testing (adonis function in the vegan $\mathrm{R}$ package).

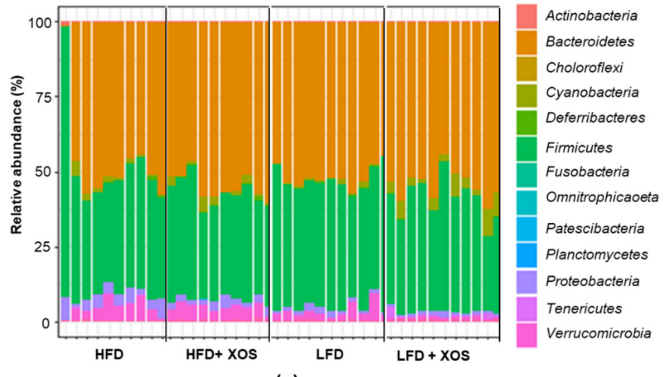

(a)
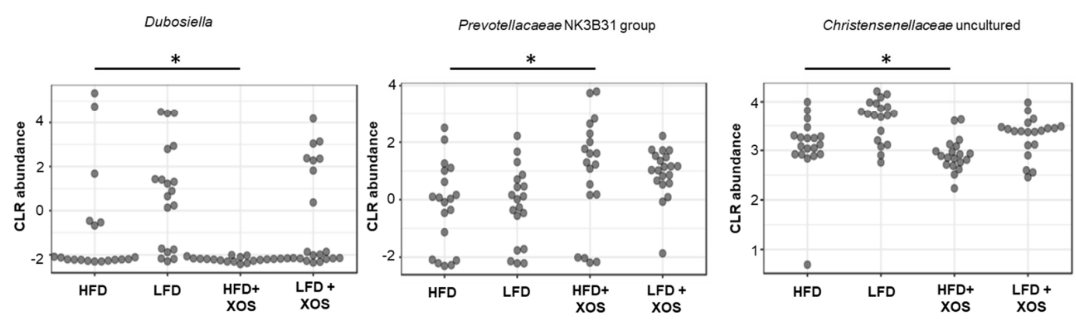

(b)

Figure 6. Differences were found in the abundance of three gut microbiota genera between the diet groups. (a) The gut microbiota composition of the rats at phylum level did not differ between the groups. $n=10 /$ diet group. The taxonomic groups were compared based on DESeq 2 using phyloseq $\mathrm{R} /$ Bioconductor package including Benjamini-Hochberg correction for multiple testing. (b) Differences between the groups were found in the relative abundance of Dubosiella, Prevotellaceae NK3B31 and uncultured genus of Christensenellaceae family. $n=10$ /diet group. The taxonomic groups were compared based on DESeq2 using phyloseq R/Bioconductor package including Benjamini-Hochberg correction for multiple testing. CLR abundance $=$ centered log-ratio transformed abundance. ${ }^{*}$ indicates statistically significant difference between the groups. 


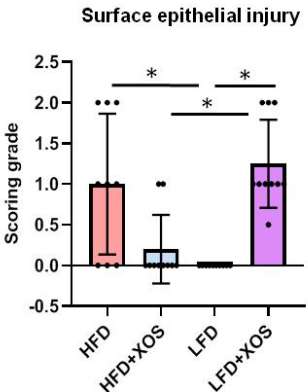

(a)

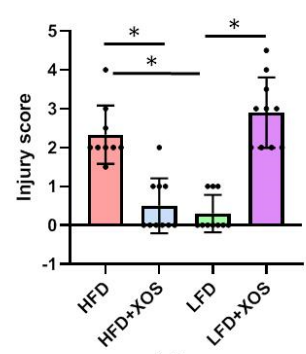

(d)

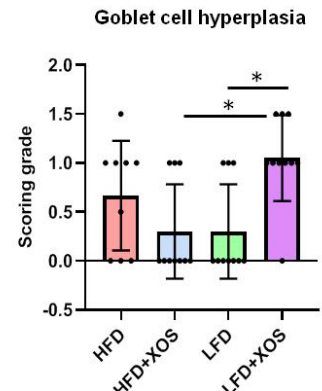

(b)

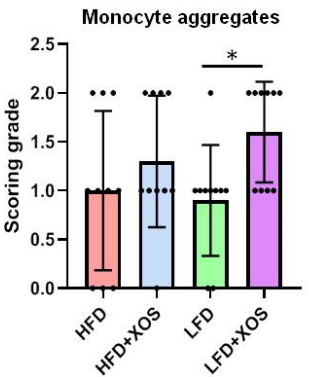

(e)
Crypt length distortion

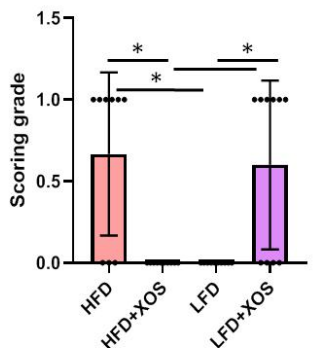

(c)

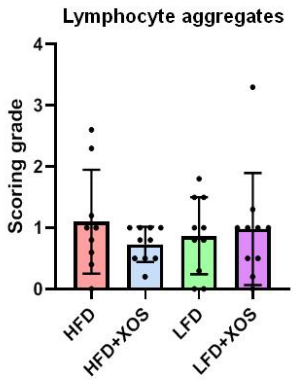

(f)

Figure 7. XOS improved the HFD-induced injury and inflammatory markers in the proximal colon. The histopathological examination detected an interactive effect of diet and XOS on the (a) surface epithelial injury; (b) Goblet cell hyperplasia; (c) crypt length distortion and; (d) injury score; (e) On the LFD, XOS increased monocyte aggregates; (f) The diets did not affect the lymphocyte aggregates. $n=8-10$ /diet group. The different data points are shown with black dots in all graphs. * denotes statistically significant difference between the groups in all graphs.
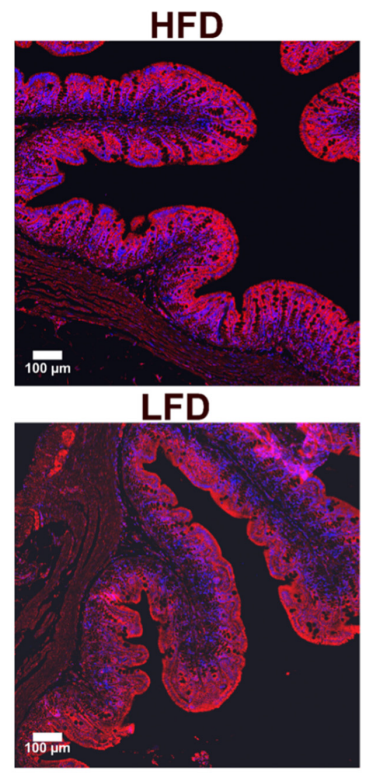

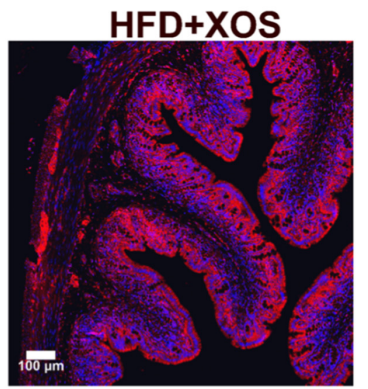

LFD+XOS

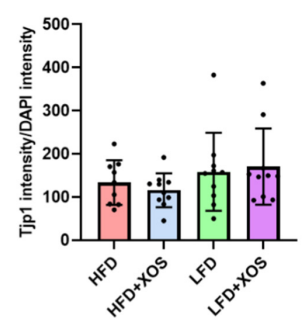

(b)

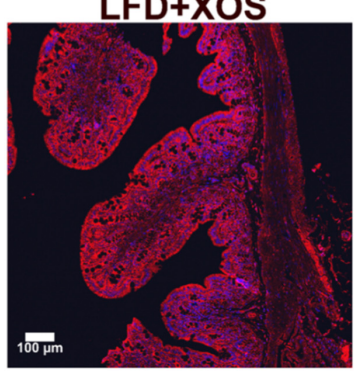

(a)

Figure 8. The intestinal Tjp1 did not differ between the diet groups. (a) The histological images were taken with Zeiss laser scanning microscopy (LSM) 700 and 20 x Plan-Apochromat 20 x/0.8 M27 objective. Tjp1 is shown with red label and $4^{\prime}, 6$-diamidino-2-phenylindole (DAPI) in blue. The scale bar is $100 \mu \mathrm{M}$; (b) The bars in the graph represent the expression of Tjp1 counted as its intensity using Image J adjusted to the intensity of DAPI. $n=9-10 /$ diet group. The different data points are shown with black dots. 
As mentioned, F. prausnitzii is reported to exert anti-inflammatory functions, for which we were interested in studying whether its increased abundance was associated with decreased systemic inflammation. Of the nine cytokines that were analyzed, only three were detected in rats, namely interleukin(IL)-10, IL-12 and tumor necrosis factor- $\alpha$ (TNF $\alpha)$. XOS supplementation and thus higher abundance of F. prausnitzii were not associated with changes in the levels of these cytokines (Figure 9). TNF $\alpha$ was under the detection limit, i.e., $<2.72 \mathrm{pg} / \mathrm{mL}$ in the HFD groups, and was detected in three out of ten rats in the LFD groups. Thus, the effect of diet on TNF $\alpha$ was not definable. The HFD diminished the serum level of anti-inflammatory IL-10 $(\mathrm{F}(1,33)=18.0, p<0.001)$ (Figure 9). Due to the importance of pathways that activate IL1 family cytokines in the development of non-alcoholic fatty liver disease (NAFLD), we also analyzed the expression of IL1 $\beta$ mRNA in the liver. No effects of the diets were found on the mRNA expression (Figure 9).

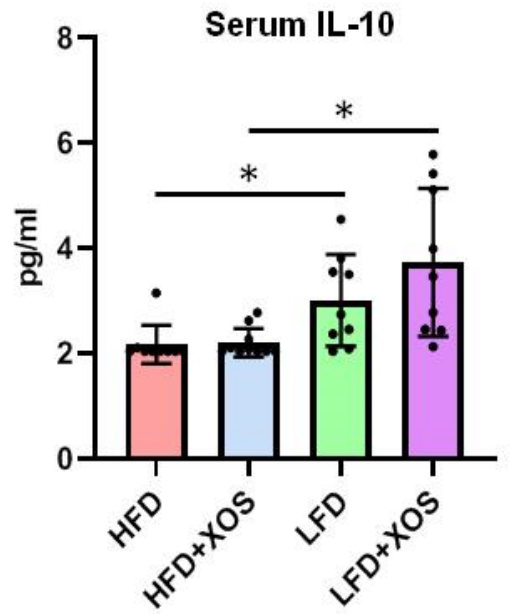

(a)

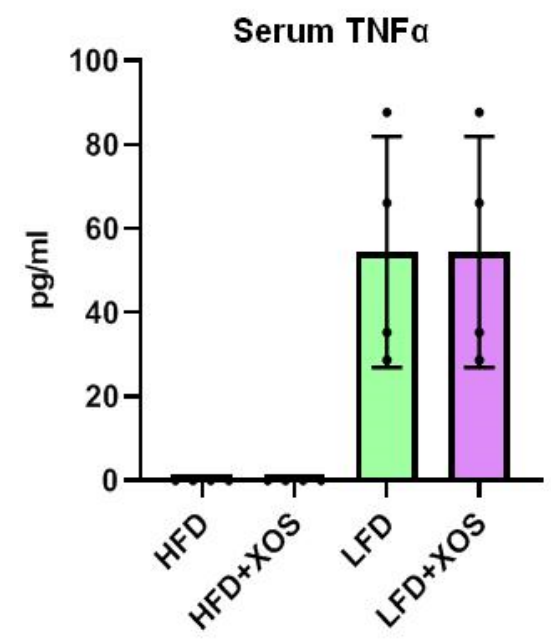

(c)

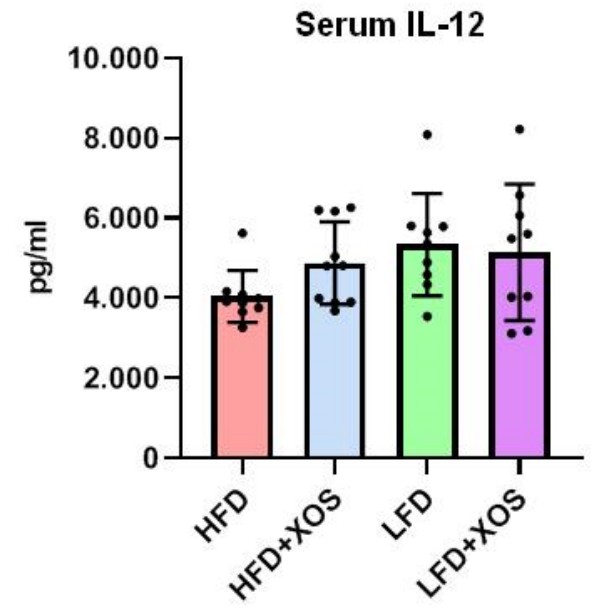

(b)

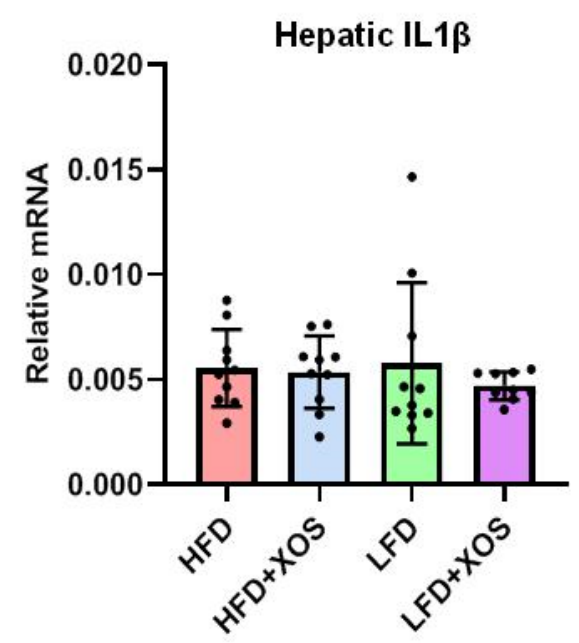

(d)

Figure 9. XOS supplementation did not affect the serum levels of (a) IL-10; (b) IL-12 or; (c) TNF $\alpha$ or; (d) the hepatic IL1 $\beta$ mRNA but the LFD groups had higher levels of anti-inflammatory IL-10. The serum cytokines were analyzed after 12 weeks of diet intervention using 9-plex ELISA, Quansys and Q-View software. Out of nine cytokines, only the levels of IL-10, IL-12 and TNF $\alpha$ were detectable in rats. IL1 $\beta$ mRNA was analyzed from hepatic cDNA with qPCR. $n=8-10 /$ diet group. The different data points are shown with black dots in all graphs. * indicates statistically significant difference between the groups in all graphs. 
3.4. The HFD Had Harmful Effects on the Cecal SCFA Profile, and on the HFD, XOS Decreased the Levels of Cecal Isovalerate and Tyrosine

The metabolomic analyses of the cecum contents detected a clear separation between the HFD and LFD based on the component $t$ [1] in OPLS-DA (Supplementary Figure S2A). The model represented a high goodness of fit $\left(R^{2} X_{(\text {cum })}=0.552\right.$ and $\left.R^{2} Y_{(\text {cum })}=0.9\right)$ and optimum predictive ability $\left(Q^{2}=0.841\right)$. The metabolites responsible for the separation of the HFD and LFD were identified by combining the information from the S-loading plot (Figure S2B), column loading plot as well as Variable Importance in Projection (VIP) data. The details and comparisons are compiled in the Supplementary Tables S1 and S2. The unsupervised PCA did not differentiate between the metabolites of the HFD and HFD+XOS (Figure S3) or between the LFD and LFD+XOS (Figure S4). As expected, the LFD was characterized by higher cecal levels of SCFAs (Table 1). The dietary fat mostly explained the clustering of the cecal metabolites (Figure 10a). In general, XOS lowered the level of cecal tyrosine $(\mathrm{F}(1,36)=7.7, p=0.009)$ (Figure 10b). XOS and diet had an interactive effect on isovalerate $(\mathrm{F}(1,36)=6.0, p=0.012)$, and on the HFD, XOS had a significant decreasing effect on it $(\mathrm{F}(1,36)=8.2, p=0.007)$ (Figure 10b). Several cecal metabolites, including the SCFAs, associated negatively with the content of hepatic triglycerides (Figure 10c). Metabolites that are known to boost oxidative metabolism, such as nicotinate, butyrate and 2-oxoglutarate, associated positively with hepatic oxidative phosphorylation and negatively with triglyceride content (Figure 10c). The group-wise comparisons of the metabolites are shown in Figure S5.

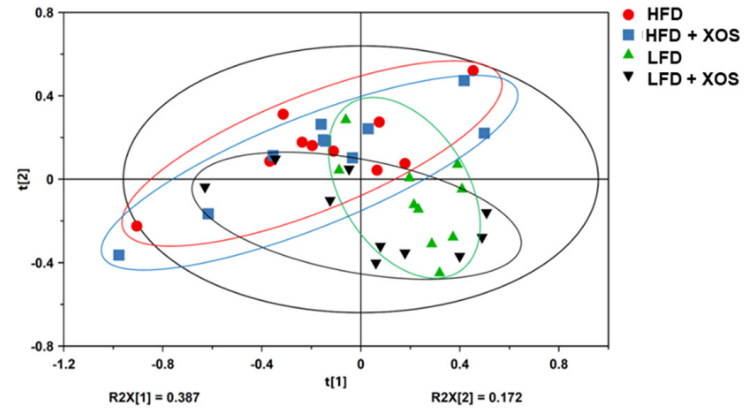

(a)
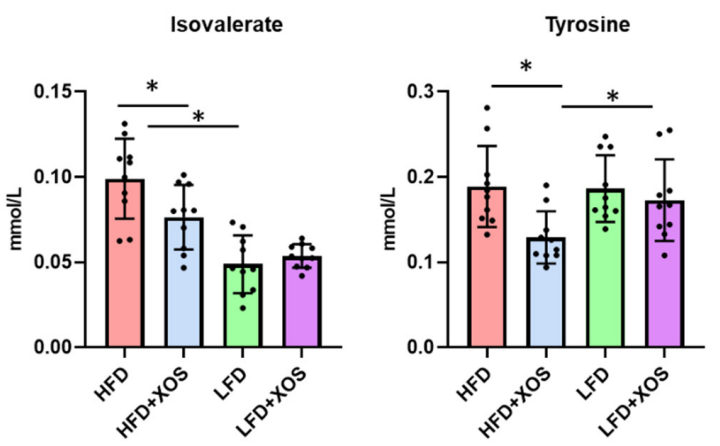

(b)

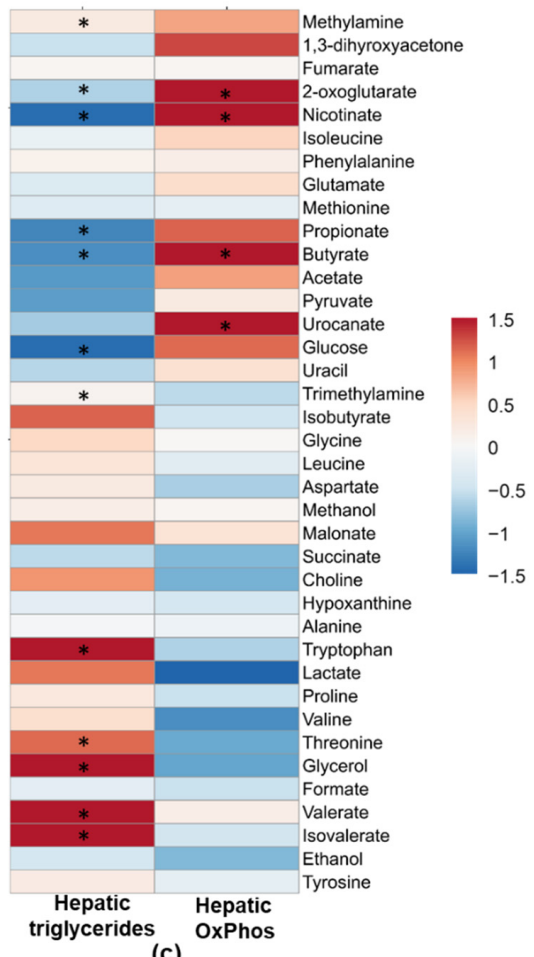

(c)

Figure 10. XOS decreased the levels of cecal tyrosine and isovalerate on the HFD. (a) The score scatter plot of the Principal Component Analysis (PCA) of the cecal metabolites showed no major differences between the diet groups. $n=10$ /diet group; (b) The levels of cecal tyrosine and isovalerate differed between the diet groups. $n=10$ /diet group. The different data points are shown with black dots. * indicates significant difference between the groups; (c) The associations of the metabolites with the hepatic triglyceride content and oxidative phosphorylation. $n=10 /$ diet group. The heatmap was drawn using Clustvis, which is a web tool used to visualize clustering of multivariate data (https://biit.cs.ut.ee/clustvis/). However, the clustering is not shown here. ${ }^{*}$ indicates significant association between the variables. The colored scale bar for the associations is shown on the right, and the color corresponds to the value of Spearman's correlation coefficient. 
Table 1. The concentrations of SCFA ( $\mathrm{mM}$, mean \pm SEM) and acetate (A): propionate (P): butyrate (B) ratio in the cecum of the rats.

\begin{tabular}{ccccc}
\hline $\begin{array}{c}\text { Group } \\
(\boldsymbol{n}=\mathbf{1 0} / \text { Group})\end{array}$ & Acetate (A) & Propionate (P) & Butyrate (B) & Ratio A:P:B \\
\hline HFD & $16 \pm 1.2$ & $3.4 \pm 0.22$ & $0.91 \pm 0.16$ & $79: 16: 5$ \\
HFD+XOS & $16 \pm 1.4$ & $3.4 \pm 0.29$ & $0.96 \pm 0.13$ & $79: 16: 5$ \\
LFD & $22 \pm 0.99$ & $5.2 \pm 0.22$ & $2.6 \pm 0.39$ & $74: 17: 9$ \\
LFD+XOS & $20 \pm 1.2$ & $4.9 \pm 0.26$ & $3.0 \pm 0.41$ & $72: 17: 11$
\end{tabular}

SCFA, short-chained fatty acids; SEM, standard error of means; XOS, xylo-oligosaccharides HFD, high-fat diet, LFD, low-fat diet.

\subsection{The HFD Decreased Energy Expenditure in Rats While the Diet and XOS Had an Interactive Effect on the Energy Expenditure}

The HFD lowered the average energy expenditure independently of the time of day (main effect of diet: daytime, $\mathrm{F}(1,36)=19.5, p<0.001$; night, $\mathrm{F}(1,35)=20.9, p<0.001$; Figure 11a). XOS and diet had an interactive effect on the nighttime lowest resting energy expenditure $(F(1,35)=7.9, p=0.008)$ and the tendency was similar during daytime $(\mathrm{F}(1,36)=3.5, p=0.071$, Figure 11a). That is, at night, HFD lowered resting energy expenditure but XOS enhanced it, whereas on the LFD, XOS diminished the resting energy expenditure. Compared with the HFD, LFD had higher measured lowest diurnal energy expenditure over $30 \mathrm{~min}(p=0.001$, Figure 11a). At nighttime, the LFD had higher average energy expenditure than the HFD ( $p=0.01$, Figure 11a). Consequently, the mean produced $\mathrm{CO}_{2}$ was higher in the LFD than the HFD during daytime $(p<0.001)$ and at night $(p=0.001$, Figure 11b). The HFD diminished the production of $\mathrm{CO}_{2}$ at nighttime $(\mathrm{F}(1,36)=198.5, p<0.001)$ and during daytime $(\mathrm{F}(1,34)=164.7, p<0.001$ Figure $11 \mathrm{~b})$. Further, the diet had a significant effect on all measured diurnal and nocturnal RQs, the RQ values being higher in the LFD than in the HFD groups. The group differences are shown in Figure 11c. In addition, the interactive effect of XOS and diet was significant on RQs at daytime and nighttime ( $p<0.001$ for all, Figure 11c).

\subsection{XOS Did Not Affect Typical NAFLD-Associated Markers or Body Composition but Had Interactive Effects on the Energy Intake}

XOS did not affect serum levels of triglycerides, free fatty acids, total cholesterol, LDL, HDL, glycerol, AST or ALT (Table 2). The diet had an effect on the serum glycerol, AST and ALT ( $p<0.001$ in each), the HFD increasing their levels. The HFD also tended to increase free fatty acids $(F(1,36)=3.8$, $p=0.059)$, while XOS tended to increase triglycerides $(F(1,35)=3.2, p=0.083)$. The LFD groups weighed less than the HFD groups during the diet intervention (Figure S6). XOS did not influence body fat \%, total fat mass, total tissue mass or the amount of epididymal or mesenteric fat (Figure S7). XOS enhanced the daily energy intake on the HFD and diminished the energy intake on the LFD $(p=0.002)$ (Figure S8).

We also analyzed the expression of adiponectin and leptin mRNA in the mesenteric and epididymal adipose tissues because they have been previously linked to NAFLD. The diet or XOS had no main effects on the expression of adiponectin (AdipoQ) mRNA in the mesenteric adipose tissue, but on the LFD, XOS increased its expression $(F(1,34)=4.6, p=0.04)$ (Figure 12). Leptin could not be faithfully detected from the mesenteric adipose tissue. In the epididymal adipose tissue, the diet but not XOS had an effect the expression of leptin $(\mathrm{F}(1,36)=25.5, p<0.001)$ and AdipoQ $(\mathrm{F}(1,36)=8.9, p=0.005)$ mRNA, the HFD diminishing the expression of both (Figure 12). 
Table 2. Serum clinical variables after the 12 weeks of diet intervention. The values are presented as mean \pm standard deviation (SD). $n=8-10 /$ diet group.

\begin{tabular}{ccccc}
\hline Serum Variable & $\begin{array}{c}\text { HFD } \\
\text { Mean } \pm \text { SD }\end{array}$ & $\begin{array}{c}\text { HFD }+ \text { XOS } \\
\text { Mean } \pm \text { SD }\end{array}$ & $\begin{array}{c}\text { LFD } \\
\text { Mean } \pm \text { SD }\end{array}$ & $\begin{array}{c}\text { HFD+XOS } \\
\text { Mean } \pm \text { SD }\end{array}$ \\
\hline Trigly $(\mathrm{mmol} / \mathrm{L})$ & $2.50 \pm 0.59$ & $3.00 \pm 0.95$ & $2.27 \pm 0.84$ & $2.68 \pm 0.74$ \\
FFA $(\mu \mathrm{mol} / \mathrm{L})$ & $351 \pm 158$ & $370 \pm 127$ & $281 \pm 153$ & $288 \pm 132$ \\
Chol $(\mathrm{mmol} / \mathrm{L})$ & $3.04 \pm 0.25$ & $2.85 \pm 0.31$ & $2.80 \pm 0.47$ & $2.84 \pm 0.59$ \\
LDL $(\mathrm{mmol} / \mathrm{L})$ & $0.52 \pm 0.17$ & $0.42 \pm 0.20$ & $0.45 \pm 0.16$ & $0.46 \pm 0.19$ \\
HDL $(\mathrm{mmol} / \mathrm{L})$ & $1.76 \pm 0.16$ & $1.60 \pm 0.41$ & $1.81 \pm 0.38$ & $1.62 \pm 0.35$ \\
Glycerol $(\mu \mathrm{mol} / \mathrm{L})$ & $292 \pm 59$ & $313 \pm 62$ & $190 \pm 40$ & $196 \pm 64$ \\
ALT $(\mathrm{U} / \mathrm{L})$ & $35.90 \pm 4.53$ & $37.20 \pm 4.52$ & $22.78 \pm 4.12$ & $21.60 \pm 3.20$ \\
AST $(\mathrm{U} / \mathrm{L})$ & $68.10 \pm 5.36$ & $71.67 \pm 7.68$ & $65.90 \pm 12.62$ & $57.89 \pm 10.42$ \\
Insulin $(\mathrm{iU} / \mathrm{L})$ & $2.50 \pm 0.95$ & $2.00 \pm 0.51$ & $2.30 \pm 0.70$ & $2.17 \pm 0.64$ \\
Glucose $(\mathrm{mmol} / \mathrm{L})$ & $14.30 \pm 2.79$ & $14.85 \pm 2.49$ & $13.17 \pm 3.31$ & $12.04 \pm 2.13$ \\
\hline
\end{tabular}

Abbreviations: Trigly, triglycerides; FFA, free fatty acids; Chol, total cholesterol; LDL, low-density lipoprotein cholesterol; HDL, high-density lipoprotein cholesterol; AST, aspartate aminotransferase; ALT, alanine aminotransferase; $\mathrm{SD}$, standard deviation.
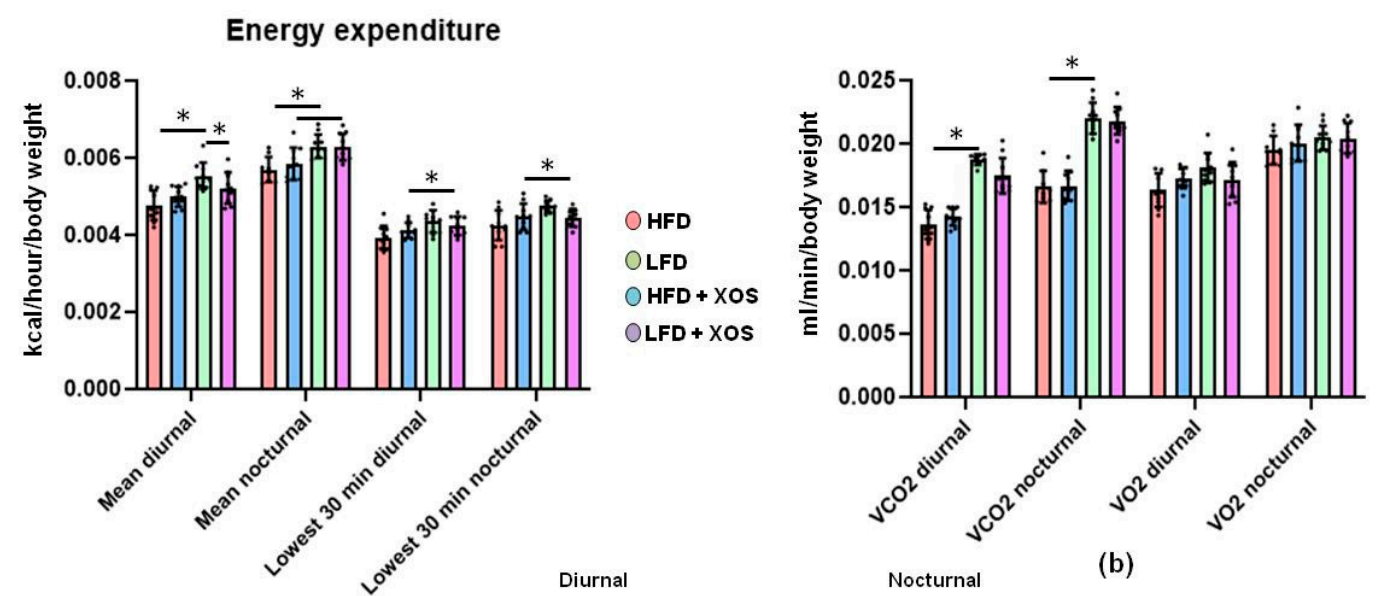

(a)

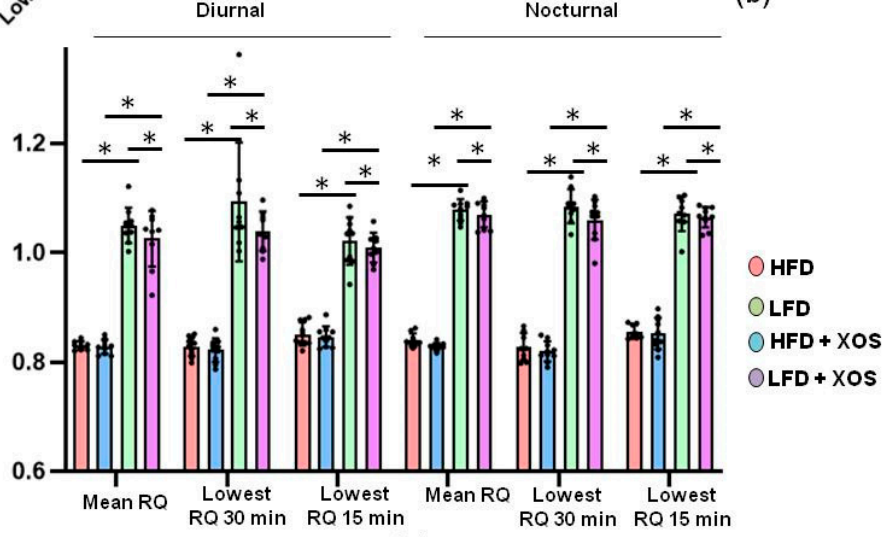

(c)

Figure 11. The LFD increased the energy expenditure in rats, while XOS and diet had an interactive effect on the respiratory quotient. (a) The average hourly energy expenditure was highest at nighttime in the LFD and the lowest measured energy expenditure was highest in the LFD at daytime; (b) The HFD diminished the production of $\mathrm{CO}_{2}$ at daytime, whereas in $\mathrm{O}_{2}$ consumption, no differences between the groups were found; (c) The respiratory quotient (RQ) values were calculated as $\mathrm{VCO}_{2} / \mathrm{VO}_{2}$. The different data points are shown with black dots. * indicates statistically significant difference between the groups. 


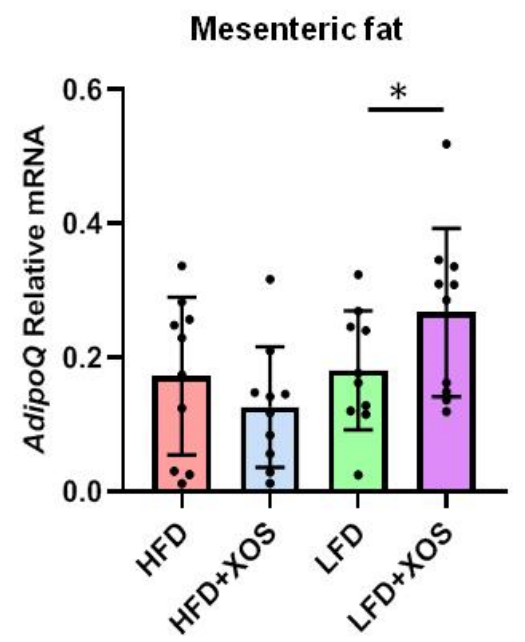

(a)

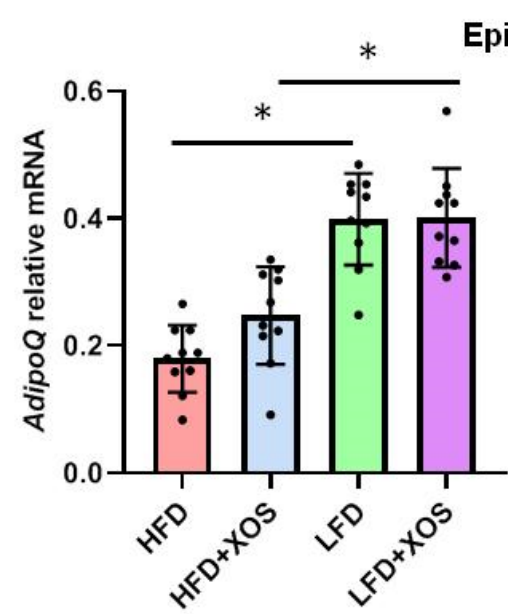

(b)

pididymal fat

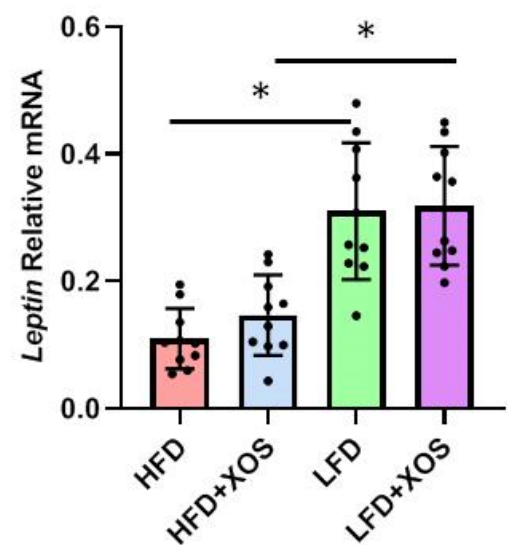

(c)

Figure 12. On the HFD, XOS did not affect the expression of leptin or adiponectin mRNA. The diet or XOS had no main effects on the expression of (a) adiponectin (AdipoQ) mRNA in the mesenteric adipose tissue, but on the LFD, XOS had an increasing effect on its expression. In the epididymal adipose tissue the diet but not XOS had an effect the expression of (b) leptin and; (c) AdipoQ mRNA. $n=8-10$ /diet group. The different data points are shown with black dots in all graphs. * indicates statistically significant difference between the groups in all graphs.

\section{Discussion}

In this study, we show that high-fat-diet-induced NAFLD in rats could be partly treated with dietary, prebiotic xylo-oligosaccharides (XOS). We show that the prebiotic diet had only minor effects on the GM in general. However, XOS slightly increased the abundance of Faecalibacterium prausnitzii, whose low relative abundance we have previously found to be associated with high hepatic fat content in humans [24]. Further, F. prausnitzii is being considered as one potential next-generation probiotic bacterium [37]. Here, we challenged the rats with high-fat diet or not, and with XOS supplementation or not. The hepatic fat and triglyceride contents were diminished in the XOS-supplemented high-fat diet group, by the XOS enhancing hepatic $\beta$-oxidation and mitochondrial respiration. However, no severe hepatic fibrosis was detected in any of the diet groups; thus, the steatosis was not in a very advanced state. Our own previous study in a mice model suggested that NAFLD could be alleviated by administering F. prausnitzii intragastrically [23]. Because not all potentially "therapeutic" bacteria are 
accepted for human use, it is important to find dietary approaches that yield effects similar to the bacteria, as it was done in the present study.

Previously, XOS has been shown to have prebiotic properties by increasing the abundance of beneficial bacteria, such as Lactobacilli and Bifidobacteria as well as Faecalibacterium species [38]. However, F. prausnitzii was not explicitly studied. In contrast to our present findings, by studying bacteria in a human colonic simulator, Christophersen et al. found that XOS decreased the growth of F. prausnitzii [28]. However, there are methodological differences between the latter and our study that may explain the controversies. Christophersen et al. first stimulated the GM with 3\% soy protein to study its detrimental effects on the gut health. Then, the treatment was followed by soy protein with $1 \%$ XOS.

In the present study, XOS improved the HFD-induced colonic injury score and crypt length distortion. Our findings are in accordance with a previous study showing that prebiotic oligosaccharides can prevent HFD-induced intestinal damages [39]. However, XOS did not affect intestinal Tjp1 according to our immunohistological analysis. The latter finding was somewhat surprising because we earlier showed that F. prausnitzii administration increased the expression of intestinal Tjp1 mRNA [23], and others have shown that prebiotic oligosaccharides in general can prevent HFD-induced impairment of gut permeability [39]. Thus, further studies are needed to determine the effects of XOS on other tight junction proteins.

Independent of the dietary fat, XOS increased the abundance of Prevotellacaeae NK3B31 group in rats. Similarly, others have shown that by increasing the amount of dietary fiber by pectin supplementation, the abundance of this bacterial group increased in rats [40]. To our knowledge, Prevotellacaeae NK3B31 has not been linked before our study to NAFLD. However, it has been shown to associate negatively with serum triglycerides and LDL cholesterol in type 2 diabetic rats [40]. Dubosiella and uncultured members of Christensenellaceae were as high in the LFD groups as in the HFD group without XOS supplementation. These were rather unexpected findings, because an obese human cohort has been characterized by a decreased relative abundance of Christensenellaceae [41], and a member of this family, Christensenella minuta, has been suggested to promote a lean host phenotype [42]. Interestingly, in our study, XOS supplementation decreased the abundance of Christensenellaceae in the HFD group that had higher body weight.

Despite the increase in the abundance of $F$. prausnitzii, which is considered an important anti-inflammatory bacterium [21,22], no differences in the levels of serum cytokines IL-10, IL-12 or TNF $\alpha$ were found in response to the XOS supplementation. However, it should be noted that we determined the levels of the cytokines only at the end of the diet intervention; thus, we may have missed whether their concentrations were elevated at some other time point during the 12-week diet intervention. Nevertheless, the HFD lowered serum levels of anti-inflammatory IL-10. This result is in agreement with the lower levels of IL-10 in NAFLD patients than in healthy controls [43]. Regardless of the systemic cytokines, XOS seemed to reduce intestinal inflammation because it reversed the effects of the HFD on the distorted colonic crypt length, which is commonly observed in intestinal inflammation [44]. However, further studies are needed to understand why XOS on the LFD caused injuries similar to the HFD. This is surprising because XOS is known to be safe and well tolerated by normal human populations.

Of the prebiotic fibers, alpha-galacto-oligosaccharides [45] and fructo-oligosaccharides [46] have been shown to ameliorate NAFLD in preclinical animal models, but to the best of our knowledge, this study is the first to describe such an effect for XOS. Concomitantly with the increase in F. prausnitzii, XOS decreased the content of hepatic triglycerides in HFD-fed rats. This was explained by the XOS enhancing the activity of hepatic $\beta-\mathrm{HAD}$, mitochondrial respiratory capacity available for the production of ATP through the electron flow from complex I, coupling of electron transport through the complex I and oxidative phosphorylation. $\beta$-HAD is a subunit of the mitochondrial trifunctional enzyme subunit alpha (MTP), which catalyzes the last three reactions of fatty acid $\beta$-oxidation. Previously, heterozygous $\mathrm{MTP}^{+/-}$mice were shown to develop NAFLD simultaneously with a reduced rate of $\beta$-oxidation [47], thus highlighting the importance of MTP in the onset of the disease. Despite the 
improvements in hepatic metabolism, diet or XOS caused only small effects on the whole-body energy expenditure and respiratory quotient. However, as expected, the HFD enhanced the caloric intake, body weight gain and body fat content compared to the LFD. On the HFD, XOS diminished the levels of cecal metabolites tyrosine and isovalerate, whose reduction likely contributed to the increased hepatic fat oxidation. We assume this because higher levels of tyrosine have been observed in NASH patients [48] and, in addition, dysregulated metabolism of tyrosine has been documented in them [49]. The consequences of the dysregulated metabolism of tyrosine are not entirely clear, but it has been proposed that tyrosine could enter the ketogenic pathway, in which it will be metabolized directly to acetyl-CoA [50]. Therefore, high levels of tyrosine might enhance the synthesis of fatty acids and thus further contribute to the deposition of lipids in liver. The GM-produced isovalerate could also have participated in the NAFLD of the rats. This view is supported by a study in which the GMs from NAFLD mice and healthy mice were transplanted into germ-free mice. The mice receiving the GM of the NAFLD mice had significantly higher fecal isovalerate levels and reproduced NAFLD [51]. In our study, fecal nicotinic acid, butyrate and 2-oxoglutarate were associated negatively with hepatic fat content and positively with oxidative phosphorylation. Behind these associations may be the capacity of nicotinic acid to inhibit the flux of fatty acids from the adipose tissue to the liver [52]. Similarly, 2-oxoglutarate and butyrate are known to promote hepatic oxidative metabolism [53].

\section{Conclusions}

Our study provides evidence that specific, prebiotic dietary supplements can be used to ameliorate NAFLD. Further, we identified the enhanced hepatic oxidative metabolism and mitochondrial functions as the underlying prebiotic-dependent preventive mechanisms of NAFLD that further may be associated with the gut microbiota. However, our study was done in rats and it should be further studied in human cohorts whether our findings on XOS improving NAFLD can be extended to humans.

Supplementary Materials: The following are available online at http://www.mdpi.com/2072-6643/12/11/3225/s1: Supplementary methods: Histopathologic grading of the proximal colon, Figure S1: The Sirius Red staining for the estimation of the amount of liver fibrosis, Figure S2: OPLS-DA of cecal metabolic profiles between the HFD and LFD, Figure S3: Score scatter plot of the Principal Component Analysis of the cecal metabolic profiles between the HFD and HFD+XOS rats. Figure S4: Multivariate analysis of the cecal metabolic profiles between the LFD and LFD+XOS rats, Figure S5: The concentrations of the cecal metabolites in different diet groups, Figure S6: Development of body weight during the diet intervention, Figure S7: XOS did not modulate body composition measured by dual energy x-ray absorption or tissue weight at necropsy, Figure S8: The energy intake during the diet intervention, Table S1: List of highly influential variables (bins) identified by combining S-plot, column loading plot and VIP values $\geq 0.7$, as obtained from the OPLS-DA, Table S2: Concentrations of the cecal metabolites that discriminated between the HFD and LFD.

Author Contributions: Conceptualization, S.L., E.M. (Eveliina Munukka), and S.P.; methodology, S.L., R.P., E.M. (Eveliina Munukka), W.S., E.M. (Elina Mäkinen), A.D., B.Y., J.L., M.L., M.T., and S.P.; formal analysis, S.L., L.L., B.Y., M.T., and S.P.; resources, L.L., M.T., and S.P.; writing-original draft preparation, S.L., L.L., and S.P.; writing-review and editing, all authors; supervision, L.L. and S.P.; funding acquisition, B.Y., M.T., L.L., and S.P. All authors have read and agreed to the published version of the manuscript.

Funding: This study was financially supported by the Academy of Finland Researcher fellowship for Pekkala (Grant ID 308042) and by the ERVA funding of The Hospital District of Southwest Finland for Pekkala. The foundation of Jenny and Antti Wihuri and the Central Finland Regional fund of the Finnish Cultural Foundation are acknowledged for their personal grants to S.L. to perform this study.

Acknowledgments: We thank Eliisa Kiukkanen for the animal care and maintenance. We thank Sanja Vanhatalo for the help in Faecalibacterium prausnitzii cultures. Anniina Keskitalo is thanked for preparing the F. prausnitzii standard curve for qPCR. We thank Mervi Matero, Hanne Tähti, Jukka Hintikka, Elina Virtanen, and Markus Honkanen for the excellent technical assistance.

Conflicts of Interest: The authors declare no conflict of interest. The funders had no role in the design of the study; in the collection, analyses, or interpretation of data; in the writing of the manuscript, or in the decision to publish the results. 


\section{References}

1. Araujo, A.R.; Rosso, N.; Bedogni, G.; Tiribelli, C.; Bellentani, S. Global epidemiology of non-alcoholic fatty liver disease/non-alcoholic steatohepatitis: What we need in the future. Liver Int. 2018, 38 (Suppl. S1), 47-51. [CrossRef] [PubMed]

2. Wong, V.W.; Wong, G.L.; Choi, P.C.; Chan, A.W.; Li, M.K.; Chan, H.Y.; Chim, A.M.; Yu, J.; Sung, J.J.; Chan, H.L. Disease progression of non-alcoholic fatty liver disease: A prospective study with paired liver biopsies at 3 years. Gut 2010, 59, 969-974. [CrossRef] [PubMed]

3. McPherson, S.; Hardy, T.; Henderson, E.; Burt, A.D.; Day, C.P.; Anstee, Q.M. Evidence of NAFLD progression from steatosis to fibrosing-steatohepatitis using paired biopsies: Implications for prognosis and clinical management. J. Hepatol. 2015, 62, 1148-1155. [CrossRef] [PubMed]

4. Tilg, H.; Moschen, A.R. Evolution of inflammation in nonalcoholic fatty liver disease: The multiple parallel hits hypothesis. Hepatology 2010, 52, 1836-1846. [CrossRef] [PubMed]

5. Ozcan, L.; Ergin, A.S.; Lu, A.; Chung, J.; Sarkar, S.; Nie, D.; Myers, M.G., Jr.; Ozcan, U. Endoplasmic reticulum stress plays a central role in development of leptin resistance. Cell Metab. 2009, 9, 35-51. [CrossRef]

6. Sha, H.; He, Y.; Chen, H.; Wang, C.; Zenno, A.; Shi, H.; Yang, X.; Zhang, X.; Qi, L. The IRE1alpha-XBP1 pathway of the unfolded protein response is required for adipogenesis. Cell Metab. 2009, 9, 556-564. [CrossRef]

7. Begriche, K.; Massart, J.; Robin, M.A.; Bonnet, F.; Fromenty, B. Mitochondrial adaptations and dysfunctions in nonalcoholic fatty liver disease. Hepatology 2013, 58, 1497-1507. [CrossRef]

8. Zmora, N.; Suez, J.; Elinav, E. You are what you eat: Diet, health and the gut microbiota. Nat. Rev. Gastroenterol. Hepatol. 2019, 16, 35-56. [CrossRef]

9. Henao-Mejia, J.; Elinav, E.; Thaiss, C.A.; Licona-Limon, P.; Flavell, R.A. Role of the intestinal microbiome in liver disease. J. Autoimmun. 2013, 46, 66-73. [CrossRef]

10. Henao-Mejia, J.; Elinav, E.; Thaiss, C.A.; Flavell, R.A. The intestinal microbiota in chronic liver disease. Adv. Immunol. 2013, 117, 73-97. [CrossRef]

11. Spencer, M.D.; Hamp, T.J.; Reid, R.W.; Fischer, L.M.; Zeisel, S.H.; Fodor, A.A. Association between composition of the human gastrointestinal microbiome and development of fatty liver with choline deficiency. Gastroenterology 2011, 140, 976-986. [CrossRef]

12. Michail, S.; Lin, M.; Frey, M.R.; Fanter, R.; Paliy, O.; Hilbush, B.; Reo, N.V. Altered gut microbial energy and metabolism in children with non-alcoholic fatty liver disease. FEMS Microbiol. Ecol. 2015, 91, 1-9. [CrossRef] [PubMed]

13. Raman, M.; Ahmed, I.; Gillevet, P.M.; Probert, C.S.; Ratcliffe, N.M.; Smith, S.; Greenwood, R.; Sikaroodi, M.; Lam, V.; Crotty, P.; et al. Fecal microbiome and volatile organic compound metabolome in obese humans with nonalcoholic fatty liver disease. Clin. Gastroenterol. Hepatol. 2013, 11, 868-875. [CrossRef] [PubMed]

14. Mouzaki, M.; Comelli, E.M.; Arendt, B.M.; Bonengel, J.; Fung, S.K.; Fischer, S.E.; McGilvray, I.D.; Allard, J.P. Intestinal microbiota in patients with nonalcoholic fatty liver disease. Hepatology 2013, 58, 120-127. [CrossRef] [PubMed]

15. Zhu, L.; Baker, S.S.; Gill, C.; Liu, W.; Alkhouri, R.; Baker, R.D.; Gill, S.R. Characterization of gut microbiomes in nonalcoholic steatohepatitis (NASH) patients: A connection between endogenous alcohol and NASH. Hepatology 2013, 57, 601-609. [CrossRef]

16. Shen, F.; Zheng, R.D.; Sun, X.Q.; Ding, W.J.; Wang, X.Y.; Fan, J.G. Gut microbiota dysbiosis in patients with non-alcoholic fatty liver disease. Hepatobiliary Pancreat. Dis. Int. 2017, 16, 375-381. [CrossRef]

17. Suzuki, T.A.; Worobey, M. Geographical variation of human gut microbial composition. Biol. Lett. 2014, 10, 20131037. [CrossRef]

18. O'Toole, P.W.; Jeffery, I.B. Gut microbiota and aging. Science 2015, 350, 1214-1215. [CrossRef] [PubMed]

19. Mariat, D.; Firmesse, O.; Levenez, F.; Guimaraes, V.; Sokol, H.; Dore, J.; Corthier, G.; Furet, J.P. The Firmicutes/Bacteroidetes ratio of the human microbiota changes with age. BMC Microbiol. 2009, 9, 123. [CrossRef]

20. Haro, C.; Rangel-Zuniga, O.A.; Alcala-Diaz, J.F.; Gomez-Delgado, F.; Perez-Martinez, P.; Delgado-Lista, J.; Quintana-Navarro, G.M.; Landa, B.B.; Navas-Cortes, J.A.; Tena-Sempere, M.; et al. Intestinal Microbiota Is Influenced by Gender and Body Mass Index. PLoS ONE 2016, 11, e0154090. [CrossRef] 
21. Miquel, S.; Leclerc, M.; Martin, R.; Chain, F.; Lenoir, M.; Raguideau, S.; Hudault, S.; Bridonneau, C.; Northen, T.; Bowen, B.; et al. Identification of metabolic signatures linked to anti-inflammatory effects of Faecalibacterium prausnitzii. MBio 2015, 6. [CrossRef] [PubMed]

22. Quevrain, E.; Maubert, M.A.; Michon, C.; Chain, F.; Marquant, R.; Tailhades, J.; Miquel, S.; Carlier, L.; Bermudez-Humaran, L.G.; Pigneur, B.; et al. Identification of an anti-inflammatory protein from Faecalibacterium prausnitzii, a commensal bacterium deficient in Crohn's disease. Gut 2016, 65, 415-425. [CrossRef] [PubMed]

23. Munukka, E.; Rintala, A.; Toivonen, R.; Nylund, M.; Yang, B.; Takanen, A.; Hanninen, A.; Vuopio, J.; Huovinen, P.; Jalkanen, S.; et al. Faecalibacterium prausnitzii treatment improves hepatic health and reduces adipose tissue inflammation in high-fat fed mice. ISME J. 2017. [CrossRef] [PubMed]

24. Munukka, E.; Pekkala, S.; Wiklund, P.; Rasool, O.; Borra, R.; Kong, L.; Ojanen, X.; Cheng, S.M.; Roos, C.; Tuomela, S.; et al. Gut-adipose tissue axis in hepatic fat accumulation in humans. J. Hepatol. 2014, 61, 132-138. [CrossRef]

25. Wong, V.W.; Tse, C.H.; Lam, T.T.; Wong, G.L.; Chim, A.M.; Chu, W.C.; Yeung, D.K.; Law, P.T.; Kwan, H.S.; Yu, J.; et al. Molecular characterization of the fecal microbiota in patients with nonalcoholic steatohepatitis-a longitudinal study. PLoS ONE 2013, 8, e62885. [CrossRef]

26. Markowiak, P.; Slizewska, K. Effects of Probiotics, Prebiotics, and Synbiotics on Human Health. Nutrients 2017, 9, 1021. [CrossRef]

27. Finegold, S.M.; Li, Z.; Summanen, P.H.; Downes, J.; Thames, G.; Corbett, K.; Dowd, S.; Krak, M.; Heber, D. Xylooligosaccharide increases bifidobacteria but not lactobacilli in human gut microbiota. Food Funct. 2014, 5, 436-445. [CrossRef]

28. Christophersen, C.T.; Petersen, A.; Licht, T.R.; Conlon, M.A. Xylo-oligosaccharides and inulin affect genotoxicity and bacterial populations differently in a human colonic simulator challenged with soy protein. Nutrients 2013, 5, 3740-3756. [CrossRef]

29. Scott, K.P.; Martin, J.C.; Duncan, S.H.; Flint, H.J. Prebiotic stimulation of human colonic butyrate-producing bacteria and bifidobacteria, in vitro. FEMS Microbiol. Ecol. 2014, 87, 30-40. [CrossRef]

30. Yang, J.; Summanen, P.H.; Henning, S.M.; Hsu, M.; Lam, H.; Huang, J.; Tseng, C.H.; Dowd, S.E.; Finegold, S.M.; Heber, D.; et al. Xylooligosaccharide supplementation alters gut bacteria in both healthy and prediabetic adults: A pilot study. Front. Physiol. 2015, 6, 216. [CrossRef]

31. Cunningham, J.J. Calculation of energy expenditure from indirect calorimetry: Assessment of the Weir equation. Nutrition 1990, 6, 222-223. [PubMed]

32. Ishak, K.; Baptista, A.; Bianchi, L.; Callea, F.; De Groote, J.; Gudat, F.; Denk, H.; Desmet, V.; Korb, G.; MacSween, R.N.; et al. Histological grading and staging of chronic hepatitis. J. Hepatol. 1995, 22, 696-699. [CrossRef]

33. Sokol, H.; Seksik, P.; Furet, J.P.; Firmesse, O.; Nion-Larmurier, I.; Beaugerie, L.; Cosnes, J.; Corthier, G.; Marteau, P.; Dore, J. Low counts of Faecalibacterium prausnitzii in colitis microbiota. Inflamm. Bowel Dis. 2009, 15, 1183-1189. [CrossRef] [PubMed]

34. Toivonen, R.V.S.; Hollmén, M.; Munukka, E.; Keskitalo, A.; Pietilä, S.; Elo, L.; Huovinen, P.; Jalkanen, S.; Pekkala, S. Vascular Adhesion Protein 1 Mediates Gut Microbial Flagellin-Induced Inflammation, Leukocyte Infiltration, and Hepatic Steatosis. Science 2019, 1, 65. [CrossRef]

35. Kelly, B.J.; Gross, R.; Bittinger, K.; Sherrill-Mix, S.; Lewis, J.D.; Collman, R.G.; Bushman, F.D.; Li, H. Power and sample-size estimation for microbiome studies using pairwise distances and PERMANOVA. Bioinformatics 2015, 31, 2461-2468. [CrossRef]

36. Love, M.I.; Huber, W.; Anders, S. Moderated estimation of fold change and dispersion for RNA-seq data with DESeq2. Genome Biol. 2014, 15, 550. [CrossRef]

37. Langella, P.; Guarner, F.; Martin, R. Editorial: Next-Generation Probiotics: From Commensal Bacteria to Novel Drugs and Food Supplements. Front. Microbiol. 2019, 10, 1973. [CrossRef]

38. Lin, S.H.; Chou, L.M.; Chien, Y.W.; Chang, J.S.; Lin, C.I. Prebiotic Effects of Xylooligosaccharides on the Improvement of Microbiota Balance in Human Subjects. Gastroenterol. Res. Pract. 2016, 2016, 5789232. [CrossRef]

39. Hamilton, M.K.; Ronveaux, C.C.; Rust, B.M.; Newman, J.W.; Hawley, M.; Barile, D.; Mills, D.A.; Raybould, H.E. Prebiotic milk oligosaccharides prevent development of obese phenotype, impairment of gut permeability, and microbial dysbiosis in high fat-fed mice. Am. J. Physiol. Gastrointest. Liver Physiol. 2017, 312, G474-G487. [CrossRef]

40. Wei, X.; Tao, J.; Xiao, S.; Jiang, S.; Shang, E.; Zhu, Z.; Qian, D.; Duan, J. Xiexin Tang improves the symptom of type 2 diabetic rats by modulation of the gut microbiota. Sci. Rep. 2018, 8, 3685. [CrossRef] 
41. Peters, B.A.; Shapiro, J.A.; Church, T.R.; Miller, G.; Trinh-Shevrin, C.; Yuen, E.; Friedlander, C.; Hayes, R.B.; Ahn, J. A taxonomic signature of obesity in a large study of American adults. Sci. Rep. 2018, 8, 9749. [CrossRef]

42. Goodrich, J.K.; Waters, J.L.; Poole, A.C.; Sutter, J.L.; Koren, O.; Blekhman, R.; Beaumont, M.; Van Treuren, W.; Knight, R.; Bell, J.T.; et al. Human genetics shape the gut microbiome. Cell 2014, 159, 789-799. [CrossRef] [PubMed]

43. Zahran, W.E.; Salah El-Dien, K.A.; Kamel, P.G.; El-Sawaby, A.S. Efficacy of Tumor Necrosis Factor and Interleukin-10 Analysis in the Follow-up of Nonalcoholic Fatty Liver Disease Progression. Indian J. Clin. Biochem. 2013, 28, 141-146. [CrossRef]

44. Gulhane, M.; Murray, L.; Lourie, R.; Tong, H.; Sheng, Y.H.; Wang, R.; Kang, A.; Schreiber, V.; Wong, K.Y.; Magor, G.; et al. High Fat Diets Induce Colonic Epithelial Cell Stress and Inflammation that is Reversed by IL-22. Sci. Rep. 2016, 6, 28990. [CrossRef] [PubMed]

45. Chappuis, E.; Morel-Depeisse, F.; Bariohay, B.; Roux, J. Alpha-Galacto-Oligosaccharides at Low Dose Improve Liver Steatosis in a High-Fat Diet Mouse Model. Molecules 2017, 22, 1725. [CrossRef] [PubMed]

46. Yao, F.; Jia, R.; Huang, H.; Yu, Y.; Mei, L.; Bai, L.; Ding, Y.; Zheng, P. Effect of Lactobacillus paracasei N1115 and fructooligosaccharides in nonalcoholic fatty liver disease. Arch. Med. Sci. 2019, 15, 1336-1344. [CrossRef]

47. Rector, R.S.; Morris, E.M.; Ridenhour, S.; Meers, G.M.; Hsu, F.F.; Turk, J.; Ibdah, J.A. Selective hepatic insulin resistance in a murine model heterozygous for a mitochondrial trifunctional protein defect. Hepatology 2013, 57, 2213-2223. [CrossRef] [PubMed]

48. Gitto, S.; Schepis, F.; Andreone, P.; Villa, E. Study of the Serum Metabolomic Profile in Nonalcoholic Fatty Liver Disease: Research and Clinical Perspectives. Metabolites 2018, 8, 17. [CrossRef]

49. Gaggini, M.; Carli, F.; Rosso, C.; Buzzigoli, E.; Marietti, M.; Della Latta, V.; Ciociaro, D.; Abate, M.L.; Gambino, R.; Cassader, M.; et al. Altered amino acid concentrations in NAFLD: Impact of obesity and insulin resistance. Hepatology 2018, 67, 145-158. [CrossRef]

50. Jin, R.; Banton, S.; Tran, V.T.; Konomi, J.V.; Li, S.; Jones, D.P.; Vos, M.B. Amino Acid Metabolism is Altered in Adolescents with Nonalcoholic Fatty Liver Disease-An Untargeted, High Resolution Metabolomics Study. J. Pediatr. 2016, 172, 14-19. [CrossRef]

51. Le Roy, T.; Llopis, M.; Lepage, P.; Bruneau, A.; Rabot, S.; Bevilacqua, C.; Martin, P.; Philippe, C.; Walker, F.; Bado, A.; et al. Intestinal microbiota determines development of non-alcoholic fatty liver disease in mice. Gut 2013, 62, 1787-1794. [CrossRef] [PubMed]

52. Linder, K.; Willmann, C.; Kantartzis, K.; Machann, J.; Schick, F.; Graf, M.; Kummerle, S.; Haring, H.U.; Fritsche, A.; Stefan, N.; et al. Dietary Niacin Intake Predicts the Decrease of Liver Fat Content During a Lifestyle Intervention. Sci. Rep. 2019, 9, 1303. [CrossRef] [PubMed]

53. Jones, J.G. Hepatic glucose and lipid metabolism. Diabetologia 2016, 59, 1098-1103. [CrossRef]

Publisher's Note: MDPI stays neutral with regard to jurisdictional claims in published maps and institutional affiliations.

(C) 2020 by the authors. Licensee MDPI, Basel, Switzerland. This article is an open access article distributed under the terms and conditions of the Creative Commons Attribution (CC BY) license (http://creativecommons.org/licenses/by/4.0/). 